\title{
COUNTING PROJECTIONS OF RATIONAL CURVES
}

\author{
MATTEO GALLET*,o AND JOSEF SCHICHO*
}

\begin{abstract}
Given two general rational curves of the same degree in two projective spaces, one can ask whether there exists a third rational curve of the same degree that projects to both of them. We show that, under suitable assumptions on the degree of the curves and the dimensions of the two given ambient projective spaces, the number of curves and projections fulfilling the requirements is finite. Using standard techniques in intersection theory and the Bott residue formula, we compute this number.
\end{abstract}

\section{INTRODUCTION}

Inspired by problems in multiview geometry concerning image-object correspondence under projections (see for example [BKH13], and [HZ04] for a general account on the topic), we consider the following question, where all varieties are complex:

given two general rational curves $C_{a} \subseteq \mathbb{P}^{a}$ and $C_{b} \subseteq \mathbb{P}^{b}$, both of degree $d \in \mathbb{N}$, and a natural number $c \in \mathbb{N}$,

find a rational curve $C_{c} \subseteq \mathbb{P}^{c}$ of degree $d$, together with two linear projections

$$
\pi_{a}: \mathbb{P}^{c} \rightarrow \mathbb{P}^{a}, \quad \pi_{b}: \mathbb{P}^{c} \rightarrow \mathbb{P}^{b}
$$

such that $\pi_{a}\left(C_{c}\right)=C_{a}$ and $\pi_{b}\left(C_{c}\right)=C_{b}$.

We are interested in counting the number of such curves and projections, when this number is finite up to projective equivalence in $\mathbb{P}^{c}$.

Because of the rationality assumption, we can interpret the curves appearing in the previous formulation as images of maps $f_{u}: \mathbb{P}^{1} \longrightarrow \mathbb{P}^{u}$ for $u \in\{a, b, c\}$. In this way, we can translate the original problem into a problem of vector spaces of polynomials of degree $d$ on $\mathbb{P}^{1}$. Since we are only interested in the images of the maps $f_{u}$, and not in the maps themselves, we need to allow possible reparametrizations, namely automorphisms of $\mathbb{P}^{1}$. Once we apply this translation, the problem becomes:

given two general vector subspaces $V_{a}, V_{b} \subseteq \mathbb{C}[s, t]_{d}$ of dimension $a+1$ and $b+1$, respectively, and a natural number $c \in \mathbb{N}$,

This version of the article has been accepted for publication, after peer review (when applicable) and is subject to Springer Nature's AM terms of use, but is not the Version of Record and does not reflect post-acceptance improvements, or any corrections. The Version of Record is available online at: https://doi.org/10.1007/s11856-020-2071-3.

* Supported by the Austrian Science Fund (FWF): W1214-N15 Project DK9 and P26607.

- Supported by the Austrian Science Fund (FWF): P25652 and Erwin Schrödinger Fellowship J4253. 
find automorphisms $\sigma \in \mathbb{P G L}(2, \mathbb{C})$ such that

$$
\operatorname{dim}\left(V_{a}+V_{b}^{\sigma}\right) \leq c+1
$$

Here $\mathbb{C}[s, t]_{d}$ is the vector space of homogeneous polynomials of degree $d$, and we denote by $V_{b}^{\sigma}$ the image of $V_{b}$ under the action of $\sigma$, which operates on polynomials by applying the change of coordinates determined by $\sigma$ to the variables.

A dimension count shows that one may expect that if $V_{a}$ and $V_{b}$ are general subspaces, and the condition

$$
(a+b-c+1)(d-c)=3
$$

holds, then there exists a finite number of automorphisms $\sigma \in \mathbb{P G L}(2, \mathbb{C})$ satisfying the requirements of the problem. In terms of the initial formulation, this means that if Equation $(*)$ holds and the curves $C_{a}$ and $C_{b}$ are general, then one may expect that there exist finitely many - up to changes of coordinates in $\mathbb{P}^{c}$ - curves $C_{c}$ and projections $\pi_{a}$ and $\pi_{b}$ sending $C_{c}$ to $C_{a}$ and to $C_{b}$, respectively.

The aim of this paper is, under the assumption that Equation $(*)$ holds and that the vector subspaces $V_{a}$ and $V_{b}$ are general,

- to prove that the number of automorphisms $\sigma \in \mathbb{P G L}(2, \mathbb{C})$ satisfying the requirements of the problem is indeed finite;

- to provide a formula for this number in terms of the parameters $a, b, c, d$.

This is the main result of our work (see Theorem 3.24):

Theorem. Let $C_{a} \subseteq \mathbb{P}^{a}$ and $C_{b} \subseteq \mathbb{P}^{b}$ be two general rational curves of degree $d$. Let $c$ be a natural number and suppose that Equation $(*)$ holds. Then there are, up to automorphisms of $\mathbb{P}^{c}$, finitely many rational curves $C_{c} \subseteq \mathbb{P}^{c}$ of degree $d$ together with linear projections $\pi_{a}: C_{c} \longrightarrow C_{a}$ and $\pi_{b}: C_{c} \longrightarrow C_{b}$.

(1) Suppose that $a+b+1-c=1$ and $d-c=3$. Then, the number of these curves and projections is

$$
\frac{1}{6}(a+3)(a+2)(a+1)(b+3)(b+2)(b+1) .
$$

(2) Suppose that $a+b+1-c=3$ and $d-c=1$. Then, the number of these curves and projections is

$$
\frac{1}{6} a b\left(a^{2}-1\right)\left(b^{2}-1\right)
$$

The paper is structured as follows. In Section 1 we operate the translation from the first to the second formulation of the problem. In Section 2 we prove that the number of solutions to our problem is finite when Equation $(*)$ holds. Eventually, in Section 3 we prove the formulas counting the number of solutions by means of intersection theory and the Bott residue formula.

\section{From Curves to LineAR Systems on $\mathbb{P}^{1}$}

As explained in the Introduction, we are given two general rational curves $C_{a} \subseteq$ $\mathbb{P}^{a}$ and $C_{b} \subseteq \mathbb{P}^{b}$, both of degree $d$, and we ask whether there exists a non-degenerate rational curve $C_{c} \subseteq \mathbb{P}^{c}$, together with linear projections to both $C_{a}$ and $C_{b}$. The 
rationality of the curves allows us to use their parametrizations in order to attack this problem. This comes at a cost: since we are only interested in the curves, we need to take into account the possibility of reparametrizations.

If for $u \in\{a, b, c\}$ the morphism $f_{u}: \mathbb{P}^{1} \longrightarrow \mathbb{P}^{u}$ is a parametrization of the curve $C_{u}$, then our problem translates to:

given two general morphisms $f_{a}: \mathbb{P}^{1} \longrightarrow \mathbb{P}^{a}$ and $f_{b}: \mathbb{P}^{1} \longrightarrow \mathbb{P}^{b}$ of degree $d$, and a number $c \in \mathbb{N}$,

find a morphism $f_{c}: \mathbb{P}^{1} \longrightarrow \mathbb{P}^{c}$, together with two linear projections $\pi_{a}, \pi_{b}$ and two isomorphisms $\sigma_{a}, \sigma_{b}: \mathbb{P}^{1} \longrightarrow \mathbb{P}^{1}$ making the following diagram commutative:

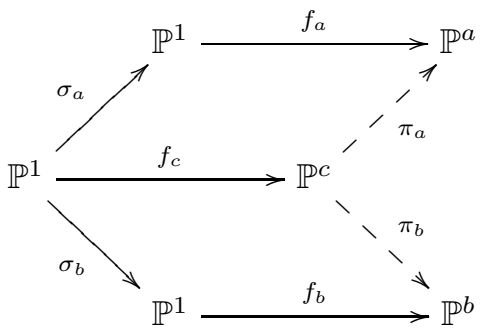

We notice that, by eventually re-defining the map $f_{c}$, we can always suppose that in Diagram (1) the map $\sigma_{a}$ is the identity, so we ask whether there exists an automorphism $\sigma \in \mathbb{P G L}(2, \mathbb{C})$ such that the following diagram commutes.

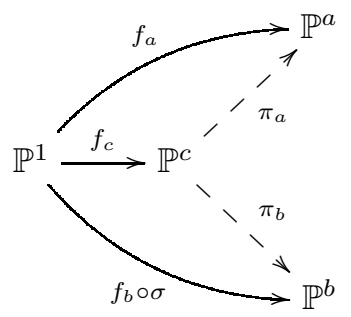

Let us write

$$
f_{a}=\left(F_{0}: \ldots: F_{a}\right), \quad f_{b}=\left(G_{0}: \ldots: G_{b}\right), \quad f_{c}=\left(H_{0}: \ldots: H_{c}\right),
$$

where $\left\{F_{i}\right\}_{i=0}^{a},\left\{G_{j}\right\}_{j=0}^{b}$ and $\left\{H_{k}\right\}_{k=0}^{c}$ are homogeneous polynomials of degree $d$ in $\mathbb{C}[s, t]$. If we denote by $G_{j}^{\sigma}$ the image of $G_{j}$ under the action of $\sigma$, then the commutativity of Diagram (2) is equivalent to

$$
\begin{aligned}
\left(F_{0}: \ldots: F_{a}\right) & =\left(\sum \lambda_{0 j} H_{j}: \ldots: \sum \lambda_{a j} H_{j}\right) \\
\left(G_{0}^{\sigma}: \ldots: G_{b}^{\sigma}\right) & =\left(\sum \mu_{0 j} H_{j}: \ldots: \sum \mu_{b j} H_{j}\right)
\end{aligned}
$$

for some complex coefficients $\left\{\lambda_{i j}\right\}_{i \in\{0, \ldots, a\}}^{j \in\{0, \ldots, c\}}$ and $\left\{\mu_{i j}\right\}_{i \in\{0, \ldots, b\}}^{j \in\{0, \ldots, c\}}$. If we define

$$
V_{a}:=\left\langle F_{0}, \ldots, F_{a}\right\rangle \text { and } V_{b}:=\left\langle G_{0}, \ldots, G_{b}\right\rangle
$$

then, taking into account Equation (3), one sees that the existence of maps $f_{c}$, projections $\pi_{a}$ and $\pi_{b}$ and automorphisms $\sigma$ as in Diagram (2) is equivalent to the existence of automorphisms $\sigma$ such that

$$
\operatorname{dim}\left(V_{a}+V_{b}^{\sigma}\right) \leq c+1
$$


where recall that we denote by $V_{b}^{\sigma}$ the image of $V_{b}$ under the action of $\sigma$, which operates by a change of coordinates.

This completes the translation of our initial problem into a question concerning vector subspaces of the space of binary homogeneous polynomials of degree $d$. Therefore from now on we will be concerned with the problem (already reported in the Introduction):

given two vector subspaces $V_{a}, V_{b} \subseteq \mathbb{C}[s, t]_{d}$ of dimension $a+1$ and $b+1$, respectively, and a natural number $c \in \mathbb{N}$,

find automorphisms $\sigma \in \mathbb{P G L}(2, \mathbb{C})$ such that

$$
\operatorname{dim}\left(V_{a}+V_{b}^{\sigma}\right) \leq c+1 .
$$

We conclude this section by mentioning a duality between instances and solutions of the previous problem that will be useful in the next sections. Consider the following perfect pairing between spaces of binary polynomials of degree $d$ :

$$
\begin{array}{ccc}
(\cdot, \cdot) & \mathbb{C}[s, t]_{d} \times \mathbb{C}[s, t]_{d} & \mathbb{C} \\
\left(p_{0} s^{d}+\cdots+p_{d} t^{d}, q_{0} s^{d}+\cdots+q_{d} t^{d}\right) & \mapsto \sum_{i=0}^{d} p_{i} q_{d-i} \frac{(-1)^{i}}{\left(\begin{array}{l}
d \\
i
\end{array}\right)}
\end{array}
$$

This is the unique (up to scaling) pairing that is invariant under the action of the group $\mathbb{P G L}(2, \mathbb{C})$ by change of coordinates. It turns out to be the $d$-th symmetric power of the pairing on the set of linear forms given by the determinant (see [Dol12, Section 1.5.1]). Using this pairing, we can define the orthogonal space to every vector subspace $V \subseteq \mathbb{C}[s, t]_{d}$ by setting

$$
V^{\perp}:=\left\{F \in \mathbb{C}[s, t]_{d}:(F, G)=0 \text { for all } G \in V\right\} .
$$

Every automorphism $\sigma \in \mathbb{P G L}(2, \mathbb{C})$ admits an adjoint $\sigma^{\perp}$ with respect to this pairing, such that $\left(F^{\sigma}, G\right)=\left(F, G^{\sigma^{\perp}}\right)$. With these definitions, one can check that there is a bijection, given by $\sigma \leftrightarrow \sigma^{\perp}$, between the sets:

(5) $\left\{\begin{array}{c}\text { automorphisms } \sigma \text { such that } \\ \operatorname{dim}\left(V_{a}+V_{b}^{\sigma}\right) \leq c+1\end{array}\right\} \longleftrightarrow\left\{\begin{array}{c}\text { automorphisms } \sigma^{\perp} \text { such that } \\ \operatorname{dim}\left(V_{a}^{\perp}+\left(V_{b}^{\perp}\right)^{\sigma^{\perp}}\right) \leq d-c-1\end{array}\right\}$

\section{Finiteness of solutions}

In this section we prove that if $V_{a}$ and $V_{b}$ are general and Equation $(*)$ holds, namely

$$
(a+b-c+1)(d-c)=3,
$$

then the number of automorphisms $\sigma \in \mathbb{P G L}(2, \mathbb{C})$ such that $\operatorname{dim}\left(V_{a}+V_{b}^{\sigma}\right) \leq$ $c+1$ is finite. To understand why we expect this result, we can argue as follows. Since $V_{a}$ has dimension $a+1$, then its orthogonal $V_{a}^{\perp}$ has dimension $d-a$. If we fix a basis $L_{1}, \ldots, L_{d-a}$ of $V_{a}^{\perp}$ and a basis $G_{0}, \ldots, G_{b}$ of $V_{b}$, then the condition $\operatorname{dim}\left(V_{a}+V_{b}^{\sigma}\right) \leq c+1$ is equivalent to imposing that the rank of the matrix with entries $\left(L_{i}, G_{j}^{\sigma}\right)$ is $c-a$, where $(\cdot, \cdot)$ is the pairing defined in Section 1. The matrix is of size $(d-a) \times(b+1)$, and so one can expect that this rank condition is a condition on $\mathbb{P G L}(2, \mathbb{C})$ of codimension (see [Har95, Proposition 12.2])

$$
((d-a)-(c-a))((b+1)-(c-a))=(d-c)(a+b-c+1) .
$$


Since $\mathbb{P G L}(2, \mathbb{C})$ is three-dimensional, if we want a finite number of solution we should suppose that the previous quantity equals 3 .

Define the incidence variety

$$
\begin{aligned}
& \mathscr{I}=\left\{\left(\sigma,\left[V_{a}\right],\left[V_{b}\right]\right) \in \mathbb{P G L}(2, \mathbb{C}) \times \mathbb{G}(a, d) \times \mathbb{G}(b, d):\right. \\
& \left.\operatorname{dim}\left(V_{a}+V_{b}^{\sigma}\right) \leq c+1\right\}
\end{aligned}
$$

where $\mathbb{G}(b, n)$ is the Grassmannian of $b$-dimensional linear subvarieties of $\mathbb{P}^{n}$, and $[\cdot]$ denotes the element in the Grassmannian determined by a vector subspace of $\mathbb{C}[s, t]_{d}$. If we consider the projection

$$
\psi: \mathscr{I} \longrightarrow \mathbb{P G L}(2, \mathbb{C}) \times \mathbb{G}(b, d)
$$

on the first and the third component, then we see that the fibers of $\psi$ are isomorphic to Schubert varieties of $\mathbb{G}(a, d)$. In fact, following [EH16, Section 4.1], if we fix $\sigma \in \mathbb{P G L}(2, \mathbb{C})$ and $\left[V_{b}\right] \in \mathbb{G}(b, d)$, we can define a complete flag

$$
\mathbb{F}: \quad\{0\} \subsetneq \mathbb{F}_{1} \subsetneq \mathbb{F}_{2} \subsetneq \cdots \subsetneq \mathbb{F}_{d+1}=\mathbb{C}[s, t]_{d}
$$

for $\mathbb{C}[s, t]_{d}$ such that $\mathbb{F}_{b+1}=V_{b}^{\sigma}$. The fiber of $\psi$ over $\left(\sigma,\left[V_{b}\right]\right)$ can be written as

$$
\psi^{-1}\left(\sigma,\left[V_{b}\right]\right)=\left\{\left(\sigma,\left[V_{a}\right],\left[V_{b}\right]\right): \operatorname{dim}\left(V_{a} \cap V_{b}^{\sigma}\right) \geq a+b-c+1\right\} .
$$

One can check that the latter is isomorphic to the Schubert variety

$$
\Sigma_{\lambda}(\mathbb{F})=\left\{\Lambda \in \mathbb{G}(a, d): \operatorname{dim}\left(\mathbb{F}_{d-a+i-\lambda_{i}} \cap \Lambda\right) \geq i \text { for all } i \in\{1, \ldots, a+1\}\right\},
$$

where

$$
\lambda=(\underbrace{d-c, \ldots, d-c}_{(a+b-c+1) \text { times }}, \underbrace{0, \ldots, 0}_{(c-b) \text { times }}) .
$$

The fibers of $\psi$ are hence irreducible; moreover, their codimension in $\mathbb{G}(a, d)$ is

$$
(d-c)(a+b-c+1)
$$

This implies that also $\mathscr{I}$ is irreducible, and a direct computation shows that if Equation $(*)$ holds, then

$$
\operatorname{dim}(\mathscr{I})=\operatorname{dim}(\mathbb{G}(a, d) \times \mathbb{G}(b, d)) .
$$

In order to prove our initial claim, it is enough to show that the projection

$$
\phi: \mathscr{I} \longrightarrow \mathbb{G}(a, d) \times \mathbb{G}(b, d)
$$

is dominant. By the properties of the dimension of the fiber of a regular map (see [Sha13, Section 6.3, Theorem 1.25]), it follows that in order to show that $\phi$ is dominant, it suffices to exhibit a single point in $\mathbb{G}(a, d) \times \mathbb{G}(b, d)$ whose preimage under $\phi$ is zero-dimensional.

Notice that Equation $(*)$ holds if and only if

$$
(* . a)\left\{\begin{array} { r } 
{ d - c = 3 } \\
{ a + b - c + 1 = 1 }
\end{array} \quad \text { or } \quad ( * . b ) \quad \left\{\begin{array}{r}
d-c=1 \\
a+b-c+1=3
\end{array}\right.\right.
$$


From now on we suppose that we are in the case prescribed by Equation (*.a), and at the end of the section we explain how to handle the situation determined by Equation (*.b).

We construct a point in $\mathbb{G}(a, d) \times \mathbb{G}(b, d)$ as follows. Define

$$
V_{a}:=\left(g_{a}\right)_{d} \quad V_{b}:=\left(g_{b}\right)_{d},
$$

where $(\cdot)_{d}$ denotes the $d$-th homogeneous component of a homogeneous ideal, and the polynomials $g_{a}$ and $g_{b}$ are any two homogeneous polynomials such that:

- $\operatorname{deg}\left(g_{a}\right)=d-a$ and $\operatorname{deg}\left(g_{b}\right)=d-b$;

- both $g_{a}$ and $g_{b}$ are squarefree;

- the cross-ratios of any four roots of $g_{a}$ or $g_{b}$ are different.

By construction, we have that $\operatorname{dim}\left(V_{a}\right)=a+1$ and $\operatorname{dim}\left(V_{b}\right)=b+1$, so $\left(\left[V_{a}\right],\left[V_{b}\right]\right)$ is a point in $\mathbb{G}(a, d) \times \mathbb{G}(b, d)$. Moreover, by the hypothesis on the cross-ratios, we see that for any fixed $\sigma \in \mathbb{P G L}(2, \mathbb{C})$, the polynomials $g_{a}$ and $g_{b}^{\sigma}$ can have at most three roots in common. We show now that with this choice of $V_{a}$ and $V_{b}$ there are finitely many $\sigma \in \mathbb{P G L}(2, \mathbb{C})$ such that $\operatorname{dim}\left(V_{a}+V_{b}^{\sigma}\right) \leq c+1$. Notice that, taking into account Equation (*.a), the latter condition is equivalent to

$$
\operatorname{dim}\left(V_{a} \cap V_{b}^{\sigma}\right) \geq a+b-c+1=1 .
$$

Moreover, the set $V_{a} \cap V_{b}^{\sigma}$ is constituted of the multiples of degree $d$ of the least common multiple of $g_{a}$ and $g_{b}^{\sigma}$. Since by Equation (*.a) we have $(d-a)+(d-b)=$ $d+3$, it follows

$$
\operatorname{deg}\left(\operatorname{lcm}\left(g_{a}, g_{b}^{\sigma}\right)\right)=d+3-\operatorname{deg}\left(\operatorname{gcd}\left(g_{a}, g_{b}^{\sigma}\right)\right) .
$$

Taking into account that by the cross-ratio hypothesis we have

$$
\operatorname{deg}\left(\operatorname{gcd}\left(g_{a}, g_{b}^{\sigma}\right)\right) \leq 3
$$

it follows that the only elements $\sigma \in \mathbb{P G L}(2, \mathbb{C})$ for which the condition $\operatorname{dim}\left(V_{a} \cap\right.$ $\left.V_{b}^{\sigma}\right) \geq 1$ is satisfied are the ones such that $g_{a}$ and $g_{b}^{\sigma}$ have exactly three roots in common. By the cross-ratio hypothesis, and by the fact that every automorphism of $\mathbb{P}^{1}$ is completely determined by the images of three projectively independent points, it follows that there are only finitely many such $\sigma$. Moreover, we can also count the number of these automorphisms $\sigma$ : each of them is specified by a pair constituted of a triple of roots of $g_{a}$ and a triple of roots of $g_{b}$, so in total they are (remember that all roots of $g_{a}$ and $g_{b}$ are distinct because of the squarefreeness hypothesis)

$$
6\left(\begin{array}{c}
d-a \\
3
\end{array}\right)\left(\begin{array}{c}
d-b \\
3
\end{array}\right)=6\left(\begin{array}{c}
a+3 \\
3
\end{array}\right)\left(\begin{array}{c}
b+3 \\
3
\end{array}\right)
$$

In the next section we prove that this is also the number when we take $V_{a}$ and $V_{b}$ to be general. This proves our initial claim when Equation (*.a) holds.

In order to obtain an example of a point in $\mathbb{G}(a, d) \times \mathbb{G}(b, d)$ whose preimage under $\phi$ is finite when Equation $(* . b)$ holds, we employ the perfect pairing (4) introduced in Section 1. The orthogonals $V_{a}^{\perp}$ and $V_{b}^{\perp}$ of the spaces $V_{a}$ and $V_{b}$ with respect to this pairing have dimension $d-a$ and $d-b$, respectively. By unraveling 
the definitions of the vector subspaces involved, and using the bijection (5), it follows that there is a bijection between the preimages of the points $\left(\left[V_{a}\right],\left[V_{b}\right]\right)$ and $\left(\left[V_{a}^{\perp}\right],\left[V_{b}^{\perp}\right]\right)$ — which belong to different Grassmannians and so appear in different instances of our problem. Moreover, if with $\left(\left[V_{a}\right],\left[V_{b}\right]\right)$ we are in the situation prescribed by Equation $(* . a)$, then with $\left(\left[V_{a}^{\perp}\right],\left[V_{b}^{\perp}\right]\right)$ we are in the situation prescribed by Equation $(* . b)$. This shows that we do not need to provide another example to ensure that also when Equation $(* . b)$ holds the map $\phi$ is dominant, and so our initial claim is proven.

\section{A FORMula FOR the NUMBer OF SOlutions}

In this section we compute the number of solutions of our problem when the two vector subspaces $V_{a}$ and $V_{b}$ are general. From the previous section we know that this number is finite when Equation $(*)$ holds.

We associate to $V_{b}$ a rational map $\Phi_{V_{b}}: \mathbb{P}^{3} \rightarrow \mathbb{G}(b, d)$ and to $V_{a}$ a Schubert variety $Z_{V_{a}}$ in $\mathbb{G}(b, d)$ so that the number we are looking for is the degree of $\Phi_{V_{b}}^{-1}\left(Z_{V_{a}}\right)$. The difficulty we encounter here in computing such degree is that $\Phi_{V_{b}}$ is not defined on the whole $\mathbb{P}^{3}$, hence we lift it to a morphism $\widetilde{\Phi}_{V_{b}}: \widetilde{\mathbb{P}^{3}} \longrightarrow \mathbb{G}(b, d)$, where $\widetilde{\mathbb{P}^{3}}$ is a blow up of $\mathbb{P}^{3}$. By doing this and using Porteous-Giambelli formula, everything is reduced to the computation of the Chern classes of a vector bundle on $\widetilde{\mathbb{P}^{3}}$. In particular, we will be interested in the degree of some polynomial combination of these Chern classes, and we will calculate these degrees via Bott residue formula.

We start with the construction of $\Phi_{V_{b}}$. Define $U$ to be the complement of the quadric $\{\alpha \delta-\beta \gamma=0\}$ in $\mathbb{P}^{3}$, where we take projective coordinates $(\alpha: \beta: \gamma: \delta)$. We identify a matrix $A=\left(\begin{array}{ll}\alpha & \beta \\ \gamma & \delta\end{array}\right)$ with the point $(\alpha: \beta: \gamma: \delta)$, so that $U$ is in bijection with $\mathbb{P G L}(2, \mathbb{C})$. The morphism $\Phi_{V_{b}}: U \longrightarrow \mathbb{G}(b, d)$ is defined as follows: to every point $A \in U$, we set $\Phi_{V_{b}}(A)$ to be the point in $\mathbb{G}(b, d)$ associated to the vector subspace $V_{b}^{\sigma}$, where $\sigma \in \mathbb{P G L}(2, \mathbb{C})$ is the automorphism corresponding to $A$. It is known (see [Arr96, Proposition 2.8]) that every morphism to a Grassmannian is completely determined by a locally free sheaf, together with a choice of a vector subspace of its global sections. In our case, the locally free sheaf $\mathscr{M}$ on $U$ determining $\Phi_{V_{b}}$ is generated by the columns of the matrix $\mathcal{M}$ with entries in $R=\mathbb{C}[\alpha, \beta, \gamma, \delta]$ obtained in the following way. Let $\left(G_{0}, \ldots, G_{b}\right)$ be a basis for $V_{b}$, then the $i$-th row of $\mathcal{M}$ is given by extracting the coefficients of $G_{i}$ (with respect to the monomial basis $s^{d}, \ldots, t^{d}$ of $\mathbb{C}[s, t]_{d}$ ) after having applied to it the change of variables

$$
\left(\begin{array}{l}
s \\
t
\end{array}\right) \mapsto\left(\begin{array}{ll}
\alpha & \beta \\
\gamma & \delta
\end{array}\right)\left(\begin{array}{l}
s \\
t
\end{array}\right) .
$$

If we denote by $M$ the graded submodule of $R(d)^{b+1}$ generated by the columns of $\mathcal{M}$, then $\mathscr{M}=\widetilde{M}$. Here $R(b)$ denotes the graded $R$-module obtained by shifting by $b$ the standard $\mathbb{Z}$-grading on $R$. The locally free sheaf $\mathscr{M}$ is then the restriction to $U$ of a coherent sheaf on $\mathbb{P}^{3}$, which we still denote by $\mathscr{M}$.

Notice that the group $\mathbb{P G L}(2, \mathbb{C})$ acts both from the left and from the right on $\mathbb{P}^{3}$ by left and right matrix multiplication, when we identify points in $\mathbb{P}^{3}$ with equivalence classes of $2 \times 2$ matrices. Both these actions induce naturally actions 
on $R(d)^{b+1}$. The right action will play a crucial in the following, in particular in the proof of Proposition 3.7 and in Propositions 3.10 and 3.12.

Lemma 3.1. The right action of $\mathbb{P G L}(2, \mathbb{C})$ on $R(d)^{b+1}$ preserves the submodule $M$.

Proof. The right action by an element of $\mathbb{P G L}(2, \mathbb{C})$ sends each of the generators of $M$ to a linear combination (with complex coefficients) of the same generators. This can be immediately seen if we write the matrix $\mathcal{M}$ as the product $\mathcal{K} \cdot \operatorname{Sym}_{d}\left(\begin{array}{ll}\alpha & \beta \\ \gamma & \delta\end{array}\right)$, where $\mathcal{K}$ is a $(b+1) \times(d+1)$ matrix with complex coefficients, while $\operatorname{Sym}_{d}(\cdot)$ denotes the $d$-th symmetric algebra functor. In this way, if $\sigma \in \mathbb{P G L}(2, \mathbb{C})$ is any element, then the generators of the module $M \cdot \sigma$ are the columns of the matrix $\mathcal{K} \cdot \operatorname{Sym}_{d}\left(\begin{array}{ll}\alpha & \beta \\ \gamma & \delta\end{array}\right) \cdot \operatorname{Sym}_{d}(\sigma)$, which are complex linear combinations of the columns of $\mathcal{M}$.

Remark 3.2. Consider the two rulings of the quadric $\{\alpha \delta-\beta \gamma=0\}$ in $\mathbb{P}^{3}$. A direct computation shows that the right action of $\mathbb{P G L}(2, \mathbb{C})$ preserves one of the two rulings as a whole, permuting the lines in this ruling, while it preserves each individual line of the other.

A nice property of the coherent sheaf $\mathscr{M}$ is that it is locally free on an open subset larger than $U$ and whose complement in $\mathbb{P}^{3}$ is constituted by disjoint lines. We prove this in Proposition 3.7. To this end, we introduce some technical tools.

Starting from $V_{b}$, we define a zero-dimensional subscheme in $\mathbb{P}^{1}$ that will be used to describe the locus in $\mathbb{P}^{3}$ where $\mathscr{M}$ is not locally free.

Definition 3.3. Let $V_{b} \subseteq \mathbb{C}[s, t]_{d}$ be a vector subspace of dimension $b+1$. Define

$$
\widehat{B}:=\left\{(s: t) \in \mathbb{P}^{1}: \text { there exists } G \in V_{b} \text { such that } \operatorname{ord}_{(s: t)} G \geq b+1\right\} .
$$

If $G_{0}, \ldots, G_{b}$ is a basis for $V_{b}$, then we have

$$
\widehat{B}=\left\{(s: t) \in \mathbb{P}^{1}:\left|\begin{array}{ccc}
\frac{\partial^{b} G_{0}}{\partial s^{b}}(s, t) & \cdots & \frac{\partial^{b} G_{0}}{\partial t^{b}}(s, t) \\
\vdots & \ddots & \vdots \\
\frac{\partial^{b} G_{b}}{\partial s^{b}}(s, t) & \cdots & \frac{\partial^{b} G_{b}}{\partial t^{b}}(s, t)
\end{array}\right|=0\right\} .
$$

Notice that the last description of $\widehat{B}$ allows to endow it with the structure of a scheme. Using Euler's identity for homogeneous polynomials, and column reduction, one sees that the determinant in Equation (7), multiplied by a suitable power of $s$, is a scalar multiple of

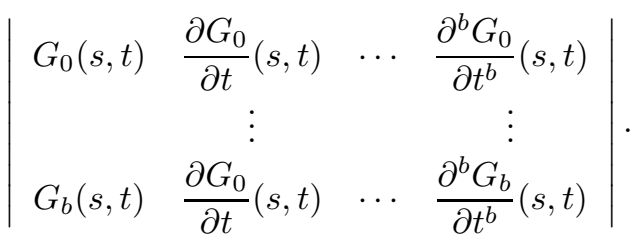

If the determinant in Equation (8) is identically zero, then in particular it is so when $s=1$. In this case, however, in Equation (8) we get the Wronskian of the polynomials $G_{0}, \ldots, G_{b}$, and the Wronskian of linearly independent polynomials 
cannot be identically zero (see $[\mathrm{BD} 10])$. Hence it cannot happen that $\widehat{B}=\mathbb{P}^{1}$. Since the determinant in Equation $(7)$ has degree $(b+1)(d-b)$, then $\widehat{B}$ is a zerodimensional scheme of length $(b+1)(d-b)$.

Lemma 3.4. Let $V_{b} \subseteq \mathbb{C}[s, t]_{d}$ be a general vector subspace of dimension $b+1$. Then the set $\widehat{B}$ as in Definition 3.3 is constituted of $(b+1)(d-b)$ distinct points.

Proof. Denote by $\operatorname{Hilb}_{(b+1)(d-b)}\left(\mathbb{P}^{1}\right)$ the Hilbert scheme of zero-dimensional schemes of length $(b+1)(d-b)$ in $\mathbb{P}^{1}$. Since it is the projectivization of the space of bivariate polynomials of degree $(b+1)(d-b)$, the scheme $\operatorname{Hilb}_{(b+1)(d-b)}\left(\mathbb{P}^{1}\right)$ is irreducible of dimension $(b+1)(d-b)$. Consider now the morphism:

$$
\begin{array}{cccc}
\Xi: \mathbb{G}(b, d) & \longrightarrow & \operatorname{Hilb}_{(b+1)(d-b)}\left(\mathbb{P}^{1}\right) \\
{\left[V_{b}\right]} & \mapsto & {[\widehat{B}]}
\end{array}
$$

where $\widehat{B}$ is as in Definition 3.3. By construction, the map $\Xi$ is a morphism between varieties of the same dimension. If we show that $\Xi$ is dominant, then the statement is proven, since the locus of schemes constituted of $(b+1)(d-b)$ distinct points is open in $\operatorname{Hilb}_{(b+1)(d-b)}\left(\mathbb{P}^{1}\right)$.

We prove that $\Xi$ is dominant as in Section 2, namely by showing that $\Xi^{-1}([Z])$ is zero-dimensional for a particular point $[Z] \in \operatorname{Hilb}_{(b+1)(d-b)}\left(\mathbb{P}^{1}\right)$. We pick $Z$ to be the subscheme in $\mathbb{P}^{1}$ defined by the ideal $\left(t^{(b+1)(d-b)}\right)$, where we take $(s: t)$ as homogeneous coordinates in $\mathbb{P}^{1}$. A direct computation shows that if we take $\bar{V}_{b}:=\left\langle s^{b} t^{d-b}, \ldots, t^{d}\right\rangle$, then $\Xi\left(\left[\bar{V}_{b}\right]\right)=[Z]$. Hence $[Z]$ is in the image of $\Xi$. Now suppose that $\Xi\left(\left[V_{b}\right]\right)=[Z]$ for some vector subspace $V_{b}=\left\langle G_{0}, \ldots, G_{b}\right\rangle$. We are going to show that $\operatorname{ord}_{(1: 0)} G_{i} \geq d-b$ for all $i \in\{0, \ldots, b\}$. If this is true, then $V_{b}$ is contained in the $d$-th homogeneous component $\left(t^{d-b}\right)_{d}$ of the ideal $\left(t^{d-b}\right)$. Since both $V_{b}$ and $\left(t^{d-b}\right)_{d}$ have dimension $b+1$, they are equal. This proves that, at least set-theoretically, the fiber $\Xi^{-1}([Z])$ is constituted of a single point, so in particular it is zero-dimensional. This concludes the proof.

To show that the order of the polynomials $\left\{G_{i}\right\}_{i=0}^{b}$ is at least $d-b$ at $(1: 0)$, let $\alpha_{i}:=\operatorname{ord}_{(1: 0)} G_{i}$ for all $i$. Notice that we can suppose that all the orders of the $G_{i}$ are different, and we can order them so that

$$
\alpha_{0} \lesseqgtr \alpha_{1} \lesseqgtr \cdots \lesseqgtr \alpha_{b} .
$$

Because of Lemma 3.5 below, we know that $\sum\left(\alpha_{i}-i\right)=(b+1)(d-b)$. This forces $\alpha_{i} \geq d-b$ for all $i$ : in fact, we have $\alpha_{b} \leq d$, so $\alpha_{b}-b \leq d-b$, hence $\alpha_{i}-i \leq d-b$ for all $i$, and so actually we must have $\alpha_{i}-i=d-b$ for all $i$.

We thank Christoph Koutschan for providing us the proof of the following lemma.

Lemma 3.5. Let $G_{0}, \ldots, G_{b}$ be homogeneous polynomials in $\mathbb{C}[s, t]_{d}$, and let $\alpha_{i}:=$ $\operatorname{ord}_{(1: 0)} G_{i}$. Suppose that $\alpha_{0} \lesseqgtr \alpha_{1} \lesseqgtr \cdots \lesseqgtr \alpha_{b}$ holds. Then the order at $(1: 0)$ of the determinant of the matrix from Equation (8) equals $\sum\left(\alpha_{i}-i\right)$.

Proof. By a direct inspection of the orders of the entries of the matrix in Equation (8), we see that the order of the determinant is at least $\sum\left(\alpha_{i}-i\right)$, and it is exactly equal to this number if no cancellation occurs when we compute the 
determinant using the standard Leibniz formula. One sees that it is harmless to set $s=1$, and that we can suppose that the coefficient of the monomial $t^{\alpha_{i}}$ in $G_{i}(1, t)$ is 1 . Everything reduces to show that the matrix obtained by taking the trailing coefficients of the entries (namely, the coefficients of the monomials where the lowest power of $t$ appears) is non-singular. This matrix is the following:

$$
\left|\begin{array}{ccccc}
1 & \alpha_{0} & \alpha_{0}\left(\alpha_{0}-1\right) & \cdots & \alpha_{0}\left(\alpha_{0}-1\right) \cdots\left(\alpha_{0}-b+1\right) \\
\vdots & \vdots & \vdots & & \vdots \\
1 & \alpha_{b} & \alpha_{b}\left(\alpha_{b}-1\right) & \cdots & \alpha_{b}\left(\alpha_{b}-1\right) \cdots\left(\alpha_{b}-b+1\right)
\end{array}\right| .
$$

By applying column reduction, the determinant in Equation (9) turns out to be equal to the Vandermonde determinant $\prod_{i<j}\left(\alpha_{i}-\alpha_{j}\right)$, which is not zero since all the $\left\{\alpha_{i}\right\}_{i=0}^{b}$ are different.

Lemma 3.6. Let $V_{b} \subseteq \mathbb{C}[s, t]_{d}$ be a general vector subspace of dimension $b+1$. Consider a point $(\bar{s}: \bar{t}) \in \mathbb{P}^{1}$ and let $G_{0}, \ldots, G_{b}$ be a basis for $V_{b}$ such that $\left.\operatorname{~ord}_{(\bar{s}: \bar{t})} G_{i}\right)_{i}$ is a strictly increasing sequence. Let $\widehat{B}$ be as in Definition 3.3. Then

- if $(\bar{s}: \bar{t}) \notin \widehat{B}$, we have $\operatorname{ord}_{(\bar{s}: \bar{t})} G_{i}=i$ for all $i$;

- if $(\bar{s}: \bar{t}) \in \widehat{B}$, we have $\operatorname{ord}_{(\bar{s}: \bar{t})} G_{i}=i$ for $i \in\{0, \ldots, b-1\}$ and $\operatorname{ord}_{(\bar{s}: \bar{t})} G_{b}=$ $b+1$.

Proof. Suppose that $(\bar{s}: \bar{t}) \notin \widehat{B}$. Then $(\bar{s}: \bar{t})$ is not a zero of the determinant in Equation (8), so the order of this determinant at $(\bar{s}: \bar{t})$ is zero. By Lemma 3.5 it follows that $\operatorname{ord}_{(\bar{s}: \bar{t})} G_{i}=i$ for all $i$.

Suppose now that $(\bar{s}: \bar{t}) \in \widehat{B}$. Then by Lemma 3.4 the determinant in Equation (8) has order 1 at $(\bar{s}: \bar{t})$. Again by Lemma 3.5 it follows that exactly one of the numbers $\left\{\operatorname{ord}_{(\bar{s}: \bar{t})} G_{i}-i\right\}_{i=0}^{b}$ equals 1 , while all the others are zero. By hypothesis we have

$$
\operatorname{ord}_{(\bar{s}: \bar{t})} G_{0}-0 \leq \operatorname{ord}_{(\bar{s}: \bar{t})} G_{1}-1 \leq \cdots \leq \operatorname{ord}_{(\bar{s}: \bar{t})} G_{b}-b,
$$

and so the only possibility is the one presented in the statement.

Proposition 3.7. For a general choice of a subspace $V_{b} \subseteq \mathbb{C}[s, t]_{d}$ of dimension $b+1$, the sheaf $\mathscr{M}$ is locally free on an open set $U^{\prime} \supseteq U$ that is the complement of $(b+1)(d-b)$ disjoint lines in $\mathbb{P}^{3}$.

Proof. We define the set

$$
B:=\left\{\left(\begin{array}{ll}
x u & x v \\
y u & y v
\end{array}\right) \in \mathbb{P}^{3}:(x: y) \in \widehat{B},(u: v) \in \mathbb{P}^{1}\right\}
$$

where $\widehat{B}$ is as in Definition 3.3. Lemma 3.4 implies that $B$ is a set of $(b+1)(d-b)$ disjoint lines in $\mathbb{P}^{3}$. In the future, we will use the fact that each of these lines is preserved by the right action of $\mathbb{P G L}(2, \mathbb{C}$ ) (see Remark 3.2 ). We define the open set $U^{\prime}$ to be the complement of $B$ in $\mathbb{P}^{3}$, so by construction we have $U \subseteq U^{\prime}$.

We prove that $\mathscr{M}$ is locally free at every point in $U^{\prime}$. As far as the points in $U$ are concerned, there is nothing to prove. Let $A \in U^{\prime} \backslash U$, then we use the left and the right action of $\mathbb{P G L}(2, \mathbb{C})$ on $\mathbb{P}^{3}$ and suppose that $A=\left(\begin{array}{ll}1 & 0 \\ 0 & 0\end{array}\right)$. Hence, it follows that with this choice of coordinates the point $(1: 0)$ does not belong to $\widehat{B}$, because 
otherwise we would have $A \in B$. Notice that the left action does not preserve the module $M$ but this is not a problem, since we only want to establish local freeness, and so we can also work with modules that are just isomorphic to $M$. By Lemma 3.6, we can choose a basis $G_{0}, \ldots, G_{b}$ of $V$ with $\operatorname{ord}_{(1: 0)} G_{i}=i$ for all $i$. In this way, the matrix $\mathcal{M}$ whose columns generate $\mathscr{M}$ has the form

$$
\mathcal{M}_{i j}=\frac{\partial^{d} G_{i}(\alpha s+\beta t, \gamma s+\delta t)}{\partial s^{d-j} \partial t^{j}}
$$

Since the question is local, we can restrict ourselves to the open chart of $\mathbb{P}^{3}$ where $\alpha=1$. This corresponds to consider $R^{\prime}=\mathbb{C}[\beta, \gamma, \delta]$, the coordinate ring of the open chart of $\mathbb{P}^{3}$ we are working on, and the restriction of $\mathscr{M}$ to such chart, whose corresponding module $M^{\prime}$ is generated by the columns of $\mathcal{M}$ where we make the substitution $\alpha=1$. Our goal is to prove that the first $b+1$ columns of $\mathcal{M}$ generate freely $M^{\prime}$ over the ring $R_{\mathscr{A}}^{\prime}$, where $\mathscr{A}$ is the maximal ideal in $R^{\prime}$ of the point $A$. To make the computations easier, we employ the substitution $s \rightarrow s-\beta t$ : such a substitution operates on the matrix $M^{\prime}$ as the multiplication on the left by an invertible $(d+1) \times(d+1)$ matrix with entries in $R^{\prime}$; hence the modules spanned by the columns of these two matrices are isomorphic. Thus, for our purposes we can suppose that the matrix $\mathcal{M}$ has the form $\mathcal{M}_{i j}=\frac{\partial^{d} G_{i}(s, \gamma s-\gamma \beta t+\delta t)}{\partial s^{d-j} \partial t^{j}}$. Eventually, we can perform the change of variables $D=\delta-\beta \gamma$, obtaining

$$
\mathcal{M}_{i j}=\frac{\partial^{d} G_{i}(s, \gamma s+D t)}{\partial s^{d-j} \partial t^{j}} .
$$

To write explicitly the entries $\mathcal{M}_{i j}$ we employ the following Taylor expansion - here we write the expansion $T(1)=T(0)+T^{\prime}(0)+\cdots$, where $T(z):=G_{i}(s, \gamma s+D t z)$ :

$$
\begin{aligned}
G_{i}(s, \gamma s+D t) & =\sum_{j=0}^{d} \frac{(D t)^{j}}{j !} \cdot \frac{\partial^{j} G_{i}}{\partial t^{j}}(s, \gamma s) \\
& =\sum_{j=0}^{d} \frac{(D t)^{j}}{j !} \cdot s^{d-j} \cdot \frac{\partial^{j} G_{i}}{\partial t^{j}}(1, \gamma) .
\end{aligned}
$$

Hence $\mathcal{M}_{i j}=\frac{D^{j}}{j !} \frac{\partial^{j} G_{i}}{\partial t^{j}}(1, \gamma)$. In particular, we see that $D^{j}$ divides $\mathcal{M}_{i j}$ for all $i, j$. Moreover, $\mathcal{M}_{00}=G_{0}(1, \gamma)$ and thus $\mathcal{M}_{00}$ does not vanish on $A$, since by hypothesis $G_{0}(1,0) \neq 0$. Therefore $\mathcal{M}_{00}$ is invertible in $R_{\mathscr{A}}^{\prime}$, so we can perform row reductions on $\mathcal{M}$ over $R_{\mathscr{A}}^{\prime}$, obtaining a new matrix $\overline{\mathcal{M}}$ whose columns generate, over $R_{\mathscr{A}}^{\prime}$, a module isomorphic to the one generated by the the columns of $\mathcal{M}$ :

$$
\begin{aligned}
& \overline{\mathcal{M}}_{0 j}:=\mathcal{M}_{0 j} \quad \text { for all } j, \\
& \overline{\mathcal{M}}_{i j}:=\mathcal{M}_{i j}-\frac{\mathcal{M}_{i 0}}{\mathcal{M}_{00}} \mathcal{M}_{0 j} \quad \text { for all } j \text {, for all } i \geq 1 \text {. } \\
& \overline{\mathcal{M}}=\left(\begin{array}{ccccc}
\overline{\mathcal{M}}_{00} & D \cdot \overline{\mathcal{M}}_{01 / D} & D^{2} \cdot \overline{\mathcal{M}}_{02} / D^{2} & \ldots & D^{d} \cdot \overline{\mathcal{M}}_{0 d} / D^{d} \\
0 & D \cdot \overline{\mathcal{M}}_{11 / D} & D^{2} \cdot \overline{\mathcal{M}}_{12} / D^{2} & \ldots & D^{d} \cdot \overline{\mathcal{M}}_{1 d} / D^{d} \\
\vdots & & & & \\
0 & D \cdot \overline{\mathcal{M}}_{b 1} / D & D^{2} \cdot \overline{\mathcal{M}}_{b 2} / D^{2} & \ldots & D^{d} \cdot \overline{\mathcal{M}}_{b d} / D^{d}
\end{array}\right) .
\end{aligned}
$$

Notice that $\overline{\mathcal{M}}_{11}=D \cdot\left(\frac{\partial G_{1}}{\partial t}(1, \gamma)-\frac{G_{1}(1, \gamma)}{G_{0}(1, \gamma)} \frac{\partial G_{0}}{\partial t}(1, \gamma)\right)$. In particular, $\overline{\mathcal{M}}_{11} / D$ does not vanish at $A$, since by hypothesis $G_{1}(1,0)=0$ and $\frac{\partial G_{1}}{\partial t}(1,0) \neq 0$. Now observe 
that the $R_{\mathscr{A}}^{\prime}$-module generated by the columns of $\overline{\mathcal{M}}$ is isomorphic to the one generated by the columns of

$$
\left(\begin{array}{ccccc}
\overline{\mathcal{M}}_{00} & D \cdot \overline{\mathcal{M}}_{01 / D} & D^{2} \cdot \overline{\mathcal{M}}_{02} / D^{2} & \ldots & D^{d} \cdot \overline{\mathcal{M}}_{0 d} / D^{d} \\
0 & \overline{\mathcal{M}}_{11 / D} & D \cdot \overline{\mathcal{M}}_{12} / D^{2} & \cdots & D^{d-1} \cdot \overline{\mathcal{M}}_{1 d} / D^{d} \\
\vdots & & & & \\
0 & \overline{\mathcal{M}}_{b 1 / D} & D \cdot \overline{\mathcal{M}}_{b 2} / D^{2} & \cdots & D^{d-1} \cdot \overline{\mathcal{M}}_{b d} / D^{d}
\end{array}\right)
$$

where we divided by $D$ all the rows from the second to the $(b+1)$-th. At this point we can repeat the Gaussian elimination using the second row, and then "divide" again by $D$ all the rows from the third to the $(b+1)$-th. The hypothesis on $A$ and on the basis $G_{0}, \ldots, G_{b}$ ensures that this process can be carried over for all rows. In this way we eventually achieve an echelonized form the matrix, which shows that the first $b+1$ columns generate freely a module isomorphic to $M^{\prime}$. Hence $M$ itself is locally free at $A$, this proving the claim.

Definition 3.8. Let $V_{a}$ be a vector subspace of $\mathbb{C}[s, t]_{d}$ of dimension $a+1$ and let $c \in \mathbb{N}$. We consider the following subvariety of the Grassmannian $\mathbb{G}(b, d)$, which by construction is a Schubert subvariety:

$$
Z_{V_{a}}:=\left\{\Lambda \in \mathbb{G}(b, d): \operatorname{dim}\left(V_{a}+\Lambda\right) \leq c+1\right\} .
$$

We are interested in the cardinality of the set $\left(\Phi_{V_{b}}\right)^{-1}\left(Z_{V_{a}}\right)$, which we proved to be finite in Section 2 when $V_{a}$ and $V_{b}$ are general. In order to compute such number, we could use the machinery of intersection theory, in particular Porteous-Giambelli theorem. Unfortunately, the domain $U$ of the regular map $\Phi_{V_{b}}$ is not a projective variety, so the result cannot be applied directly.

On the other hand, the morphism $\Phi_{V_{b}}$ gives a rational map on $\mathbb{P}^{3}$, which we still denote by $\Phi_{V_{b}}$. By what we proved in Proposition 3.7, the locus $U^{\prime}$ where $\Phi_{V_{b}}$ is regular is bigger than $U$ and it is the complement of a number of disjoint lines. However, notice that only the points in $U$ correspond to automorphisms of $\mathbb{P}^{1}$, hence they are the only ones to be considered in solving our initial problem. From the theorem of resolution of indeterminacies of a rational map (see [Cut04, Section 4.2 and Lemma 4.8]), we know that there exists a scheme $\widetilde{\mathbb{P}^{3}}$, a morphism $\tau: \widetilde{\mathbb{P}^{3}} \longrightarrow \mathbb{P}^{3}$ and a morphism $\widetilde{\Phi}_{V_{b}}: \widetilde{\mathbb{P}^{3}} \longrightarrow \mathbb{G}(b, d)$ making the following diagram commutative:

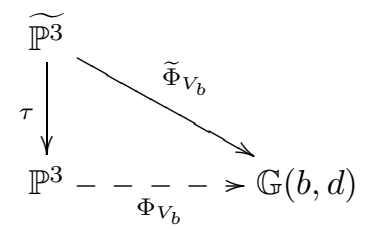

We are going to show that we can use the map $\widetilde{\Phi}_{V_{b}}$ to calculate the desired number. To do so, we have to exclude first that some point in $\widetilde{\Phi}_{V_{b}}^{-1}\left(Z_{V_{a}}\right)$ lies in $\widetilde{\mathbb{P}^{3}} \backslash \tau^{-1}(U)$, namely does not correspond to an automorphism of $\mathbb{P}^{1}$. In Lemma 3.9 we show that if $V_{a}$ and $V_{b}$ are general, then $\widetilde{\Phi}_{V_{b}}^{-1}\left(Z_{V_{a}}\right)$ is always completely contained in $\tau^{-1}(U)$. 
Lemma 3.9. Let $V_{a}, V_{b} \subseteq \mathbb{C}[s, t]_{d}$ be general vector subspaces of dimension $a+1$ and $b+1$, respectively. Suppose that Equation $(*)$ holds. Then the preimage of $Z_{V_{a}}$ under $\widetilde{\Phi}_{V_{b}}$ is contained in $\tau^{-1}(U)$.

Proof. We have to show that no element in $\widetilde{\mathbb{P}^{3}} \backslash \tau^{-1}(U)$ belongs to $\widetilde{\Phi}_{V_{b}}^{-1}\left(Z_{V_{a}}\right)$. Let $W$ be the Zariski closure of $\widetilde{P h} i_{V_{b}}\left(\widetilde{\mathbb{P}^{3}} \backslash \tau^{-1}(U)\right)$ in $\mathbb{G}(b, d)$, with the reduced structure. Notice that the irreducible components of $W$ are integral subschemes of $\mathbb{G}(b, d)$ of dimension at most 2 , since $\widetilde{\mathbb{P}^{3}} \backslash \tau^{-1}(U)$ is contained in the preimage via $\tau$ of the quadric $\{\alpha \delta-\beta \gamma=0\}$ in $\mathbb{P}^{3}$. We show that, since $V_{a}$ is general, it is always possible to avoid $W$ with $Z_{V_{a}}$. Notice that the algebraic group $\mathbb{P G L}(d+1, \mathbb{C})$ acts transitively on $\mathbb{G}(b, d)$ via its standard action on $\mathbb{P}^{d}$; if $g \in \mathbb{P G L}(d+1, \mathbb{C})$, we denote by $g \cdot Z_{V_{a}}$ the translate of $Z_{V_{a}}$ under the action of $g$. Moreover, a computation similar to the one providing Equation (6) shows that $Z_{V_{a}}$ has codimension $(d-c)(a+b-c+1)$, which equals 3 because we suppose that Equation $(*)$ holds. Then, by Kleiman's transversality theorem [Kle74, Corollary 4], the dimension of the intersection $(g$. $\left.Z_{V_{a}}\right) \cap W$ is -1 for every $g$ belonging to an open subset of $\operatorname{PGL}(d+1, \mathbb{C})$. This means that if $V_{a}$ is general, then we can suppose that $Z_{V_{a}}$ and $W$ do not intersect, and this concludes the proof.

Since, as it will be made clear in Proposition 3.10, the morphism $\tau$ is an isomorphism outside the indeterminacy locus of $\Phi_{V_{b}}$, then the cardinality of $\Phi_{V_{b}}^{-1}\left(Z_{V_{a}}\right)$ equals the cardinality of $\widetilde{\Phi}_{V_{b}}^{-1}\left(Z_{V_{a}}\right)$. Moreover, a by-product of Section 2 is that, by the fact that $V_{a}$ and $V_{b}$ are general, the fiber $\Phi_{V_{b}}^{-1}\left(Z_{V_{a}}\right)$ is constituted of smooth points. Hence, to compute the number we are interested in, it is enough to compute the degree of the 0 -cycle $\widetilde{\Phi}_{V_{b}}^{*}\left(\left[Z_{V_{a}}\right]\right)$, where $\left[Z_{V_{a}}\right]$ is the Schubert cycle given by the Schubert variety $Z_{V_{a}}$.

In order to compute this degree, let us start by noticing that we can express $\left[Z_{V_{a}}\right]$ in terms of the universal bundle $\mathscr{U}^{*}$ of the Grassmannian $\mathbb{G}(b, d)$. In fact, if $h_{1}, \ldots, h_{d-a}$ are linear forms defining $V_{a}$, we can interpret them as global sections of $\mathscr{U}^{*}$, and then $Z_{V_{a}}$ becomes the locus where the sections $h_{1}, \ldots, h_{d-a}$ have rank at most $c-a-1$ (see [Arr10, Example 4.7]). Now Schubert calculus tells us (see [Arr10, Example 4.9]) that the cycle associated to this locus is given by the determinant

$$
\left|\begin{array}{cccc}
\mathrm{c}_{(b+1)-(c-a)}\left(\mathscr{U}^{*}\right) & \mathrm{c}_{(b+2)-(c-a)}\left(\mathscr{U}^{*}\right) & \cdots & \mathrm{c}_{(b+d-a)-2(c-a)}\left(\mathscr{U}^{*}\right) \\
\mathrm{c}_{b-(c-a)}\left(\mathscr{U}^{*}\right) & \mathrm{c}_{(b+1)-(c-a-1)}\left(\mathscr{U}^{*}\right) & \cdots & \mathrm{c}_{(b+d-a)-2(c-a)+1}\left(\mathscr{U}^{*}\right) \\
\vdots & & & \\
\mathrm{c}_{b-(d-a)+2}\left(\mathscr{U}^{*}\right) & \mathrm{c}_{b-(d-a)+3}\left(\mathscr{U}^{*}\right) & \cdots & \mathrm{c}_{(b+1)-(c-a)}\left(\mathscr{U}^{*}\right)
\end{array}\right|
$$

where $\mathrm{c}_{i}\left(\mathscr{U}^{*}\right)$ denotes the $i$-th Chern class of the vector bundle $\mathscr{U}^{*}$.

Since $\widetilde{\Phi}_{V_{b}}$ is a morphism to a Grassmannian, by what we reported at the beginning of the section there exists a vector bundle $\mathscr{Q}$ on $\widetilde{\mathbb{P}^{3}}$ and a vector subspace of sections of $\mathscr{Q}$ such that the morphism they induce is $\widetilde{\Phi}_{V_{b}}$. It follows from the functoriality of the pullback that the cycle $\widetilde{\Phi}_{V_{b}}^{*}\left(\left[Z_{V_{a}}\right]\right)$ is given by the determinant in Equation (10), after substituting $\mathscr{U}^{*}$ with $\mathscr{Q}$. This is the so-called PorteousGiambelli formula, see [Arr10, Theorem 3.10] and [KL74, Corollary 6]. 
Using a "Gaussian reduction" technique as in Proposition 3.7, we can express in Proposition 3.10 the variety $\widetilde{\mathbb{P}^{3}}$ and the vector bundle $\mathscr{Q}$ in a very concrete way. We proceed following closely the technique for the resolution of indeterminacies in the case of maps to a projective space described in [Har77, Chapter II, Example 7.17.3]. Before stating the result, we need some preliminary considerations.

The right action of $\mathbb{P G L}(2, \mathbb{C})$ on $\mathbb{P}^{3}$ leaves invariant each of the $(d-b)(b+1)$ lines where the map $\Phi_{V_{b}}$ is not defined, and so by the universal property of the blowup (see [Har77, Corollary II.7.15]) it induces an action on the blowup $\mathrm{Bl}_{\mathrm{B}}\left(\mathbb{P}^{3}\right)$ of $\mathbb{P}^{3}$ at these lines. In fact $^{1}$, since $B$ is invariant under the right action of $\mathbb{P G L}(2, \mathbb{C})$, then its preimage under the map $\mathbb{P G L}(2, \mathbb{C}) \times \mathbb{P}^{3} \longrightarrow \mathbb{P}^{3}$ is $\mathbb{P G L}(2, \mathbb{C}) \times B$. Therefore, by the universal property we get a morphism $\operatorname{PGL}(2, \mathbb{C}) \times \mathrm{Bl}_{\mathrm{B}}\left(\mathbb{P}^{3}\right) \longrightarrow \mathrm{Bl}_{\mathrm{B}}\left(\mathbb{P}^{3}\right)$. By construction, for all $P \in \mathrm{Bl}_{\mathrm{B}}\left(\mathbb{P}^{3}\right) \backslash E$, where $E$ is the exceptional divisor, we have

$$
P \cdot\left(\sigma \cdot \sigma^{\prime}\right)=(P \cdot \sigma) \cdot \sigma^{\prime}, \quad P \cdot\left(\begin{array}{ll}
1 & 0 \\
0 & 1
\end{array}\right)=P \quad \text { for all } \sigma, \sigma^{\prime} \in \mathbb{P G L}(2, \mathbb{C})
$$

hence by continuity these equations hold on the whole $\mathrm{Bl}_{\mathrm{B}}\left(\mathbb{P}^{3}\right)$, thus determining the desired action.

We define a coherent sheaf $\widehat{\mathscr{Q}}$ on $\mathrm{Bl}_{\mathrm{B}}\left(\mathbb{P}^{3}\right)$ as follows. Let $\widehat{\tau}: \mathrm{Bl}_{\mathrm{B}}\left(\mathbb{P}^{3}\right) \longrightarrow \mathbb{P}^{3}$ be the blow down map. We pull back along $\widehat{\tau}$ the map $\mathscr{O}_{\mathbb{P} 3}^{d+1} \longrightarrow \mathscr{O}_{\mathbb{P} 3}(d)^{b+1}$ defined by the matrix $\mathcal{M}$. Then $\widehat{\mathscr{Q}}$ is defined as the image of this homomorphism of sheaves. It is generated by the global sections $\widehat{\tau}^{*}\left(m_{0}\right), \ldots, \widehat{\tau}^{*}\left(m_{d}\right)$, where $m_{0}, \ldots, m_{d}$ are the columns of the matrix $\mathcal{M}$. The right action of $\mathbb{P G L}(2, \mathbb{C})$ on $\mathrm{Bl}_{\mathrm{B}}\left(\mathbb{P}^{3}\right)$ preserves $\widehat{\mathscr{Q}}$. In fact, by Lemma 3.1 we have that the right action by an element $\sigma \in \mathbb{P G L}(2, \mathbb{C})$ sends each $m_{i}$ to a complex linear combination $\sum \lambda_{i j} m_{j}$. Since this holds at every point in $\mathbb{P}^{3} \backslash B$, the same is true for each $\widehat{\tau}^{*}\left(m_{i}\right)$ at every point outside the exceptional divisor. Hence, by continuity this must hold on the whole blowup, so with this action $\widehat{\mathscr{Q}}$ becomes a $\mathbb{P G L}(2, \mathbb{C})$-equivariant vector bundle.

Proposition 3.10. With the previously introduced notation, the variety $\widetilde{\mathbb{P}^{3}}$ can be taken to be the blow up of $\mathbb{P}^{3}$ at $B$, the set of $(b+1)(d-b)$ disjoint lines introduced in Proposition 3.7, with the choice of $\tau$ as the corresponding blow down morphism. With this choice $\mathscr{Q}$ is the sheaf associated to the module spanned by the pullbacks via $\tau$ of the generators of $M$.

Proof. Define $\widehat{\mathbb{P}^{3}}=\mathrm{Bl}_{\mathrm{B}}\left(\mathbb{P}^{3}\right)$ and $\widehat{\tau}: \widehat{\mathbb{P}^{3}} \longrightarrow \mathbb{P}^{3}$ to be the corresponding blow down morphism. We prove that the sheaf $\widehat{\mathscr{Q}}$ associated to the module spanned by the pullbacks of the generators of $M$ is locally free. This implies that we can take $\widehat{\mathbb{P}^{3}}=\widetilde{\mathbb{P}^{3}}, \widehat{\tau}=\tau$ and $\widehat{\mathscr{Q}}=\mathscr{Q}$, so the claim is proved. Since $\widehat{\tau}$ is an isomorphism over $U^{\prime}=\mathbb{P}^{3} \backslash B$, and $\mathscr{M}$ is locally free on $U^{\prime}$ by Proposition 3.7 , then $\widehat{\mathscr{Q}}$ is locally free on $\tau^{-1}\left(U^{\prime}\right)$. Hence we only need to check that $\widehat{\mathscr{Q}}$ is locally free on the exceptional divisors of $\widehat{\mathbb{P}^{3}}$. Since this is a local question, and all the lines in $\mathbb{P}^{3}$ forming $B$ are disjoint, we can prove the claim supposing that $B$ is constituted by a single line $L$. By a suitable change of coordinates in $\mathbb{P}^{3}$ induced by the left action

\footnotetext{
${ }^{1}$ We report here an argument by Daniel Loughran, available at https://mathoverflow.net/questions/122922/group-actions-on-blow-ups .
} 
of $\mathbb{P G L}(2, \mathbb{C})$, we can suppose that $L$ is given by $\{\gamma=\delta=0\}$. Hence $\mathrm{Bl}_{\mathrm{L}}\left(\mathbb{P}^{3}\right)$ can be written as $\operatorname{Proj}(\widetilde{R})$, with $\widetilde{R}=\mathbb{C}[\alpha, \beta, \gamma, \bar{\gamma}, \delta, \bar{\delta}, w] /(\gamma-\bar{\gamma} w, \delta-\bar{\delta} w)$, where we take the $\mathbb{Z}^{2}$-grading described by the columns of the following matrix:

$$
\left.\begin{array}{ccccccc}
\alpha & \beta & \gamma & \bar{\gamma} & \delta & \bar{\delta} & w \\
1 & 1 & 1 & 1 & 1 & 1 & 0 \\
1 & 1 & 1 & 0 & 1 & 0 & 1
\end{array}\right) .
$$

Moreover, one notices that $\widetilde{R} \cong \mathbb{C}[\alpha, \beta, \bar{\gamma}, \bar{\delta}, w]$ with the previously defined grading. The exceptional divisor $E$ of $\mathrm{Bl}_{\mathrm{L}}\left(\mathbb{P}^{3}\right)$ is then the subvariety $\{w=0\}$. We pick $P=(\alpha: \beta: \bar{\gamma}: \bar{\delta}: 0) \in E$, and we prove that $\widehat{\mathscr{Q}}$ is free at $P$. In order to simplify our computations, we consider the right action of $\mathbb{P G L}(2, \mathbb{C})$ on $\mathrm{Bl}_{\mathrm{L}}\left(\mathbb{P}^{3}\right)$, which with our choice of coordinates is given as follows: if $\left(\begin{array}{ll}u & z \\ y & v\end{array}\right)$ is an element of $\operatorname{PGL}(2, \mathbb{C})$, then its action on points of $\mathrm{Bl}_{\mathrm{L}}\left(\mathbb{P}^{3}\right)$ is given by

$$
\left(\begin{array}{c}
\alpha \\
\beta \\
\bar{\gamma} \\
\bar{\delta} \\
w
\end{array}\right) \mapsto\left(\begin{array}{c}
\alpha u+\beta y \\
\alpha z+\beta v \\
\bar{\gamma} u+\bar{\delta} y \\
\bar{\gamma} z+\bar{\delta} v \\
w
\end{array}\right)
$$

Therefore, under this action, points in the exceptional divisor of $\mathrm{Bl}_{\mathrm{L}}\left(\mathbb{P}^{3}\right)$ are equivalent to either $(1: 0: 1: 0: 0)$ or $(1: 0: 0: 1: 0)$, depending on whether $(\alpha: \beta)=(\bar{\gamma}: \bar{\delta})$ or $(\alpha: \beta) \neq(\bar{\gamma}: \bar{\delta})$ as points in $\mathbb{P}^{1}$. Let us consider the case $P=(1: 0: 1: 0: 0)$. Then the image of $P$ under the blow down map, namely $\left(\begin{array}{ll}1 & 0 \\ 0 & 0\end{array}\right)$, belongs to $B$, and this implies that $(1: 0) \in \widehat{B}$. Hence by Lemma 3.6 we are in the case where $\operatorname{ord}_{(1: 0)} G_{i}=i$ for $i \in\{0, \ldots, b-1\}$ and $\operatorname{ord}_{(1: 0)} G_{b}=b+1$. The module $\widehat{Q}$ determining $\widehat{\mathscr{Q}}$ is generated by the columns of the matrix

$$
\widehat{\mathcal{Q}}_{i j}=\frac{\partial^{d} G_{i}(\alpha s+\beta t, \bar{\gamma} w s+\bar{\delta} w t)}{\partial s^{d-j} \partial t^{j}} .
$$

Since we can take $\beta, \bar{\delta}$ and $w$ to be local coordinates for $P$, using simplifications as in the proof of Proposition 3.7 we can consider the matrix

$$
\widehat{\mathcal{Q}}_{i j}=\frac{\partial^{d} G_{i}(s, w s+w(\overbrace{\bar{\delta}-\beta}^{:=\widetilde{D}}) t)}{\partial s^{d-j} \partial t^{j}} .
$$

As in Proposition 3.7, we can expand

$$
G_{i}(s, w s+w \widetilde{D} t)=\sum_{j=0}^{d} \frac{(w \widetilde{D} t)^{j}}{j !} s^{d-j} \frac{\partial^{j} G_{i}}{\partial t^{j}}(1, w) .
$$

We can perform the Gaussian elimination that was employed in Proposition 3.7. The difference is that, here, at the $k$-th iteration of the elimination we can divide each row from the $(k+1)$-th to the $(b+1)$-th by $w \widetilde{D}$; moreover, in this case the Gaussian elimination can be performed only until the last-but-one row because of 
the orders of the polynomials $G_{i}$. The matrix we obtain has the following shape

$$
\left(\begin{array}{ccccccc}
\overline{\mathcal{Q}}_{00} & w \widetilde{D} \cdot * & w^{2} \widetilde{D}^{2} * * & \ldots & & \\
0 & \overline{\mathcal{Q}}_{11} & w \widetilde{D} \cdot * & w^{2} \widetilde{D}^{2} \cdot * & \ldots & & \\
\vdots & 0 & \ddots & \ddots & & & \\
\vdots & \vdots & & \overline{\mathcal{Q}}_{b-1, b-1} & w \widetilde{D} \cdot * & \ldots & \\
0 & 0 & \ldots & 0 & \overline{\mathcal{Q}}_{b b} & w \widetilde{D} \cdot * & \ldots
\end{array}\right)
$$

where the elements $\overline{\mathcal{Q}}_{00}, \ldots, \overline{\mathcal{Q}}_{b-1, b-1}$ are invertible in the local ring at $P$, while this is not the case for $\overline{\mathcal{Q}}_{b b}$. However, since ord $(1: 0) G_{b}=b+1$, we have that $\overline{\mathcal{Q}}_{b b}=w \cdot \overline{\overline{\mathcal{Q}}}_{b b}$ for some invertible element $\overline{\overline{\mathcal{Q}}}_{b b}$. This implies that the ideal generated by the last entries of the columns from the $(b+1)$-th to the $(d+1)$-th is principal, and so the module spanned by the columns of $\widehat{\mathcal{Q}}$ is free at $P$.

The case when $P=(1: 0: 0: 1: 0)$ can be treated in an analogous way.

The following proposition describes the class in the Chow ring of the subvariety we are interested in in terms of the Chern classes of the vector bundle $\mathscr{Q}$.

Proposition 3.11. Let $V_{a}$ and $V_{b}$ be general vector subspaces of $\mathbb{C}[s, t]_{d}$ of dimension $a+1$ and $b+1$, respectively. Suppose that

$$
(a+b-c+1)(d-c)=3 .
$$

Let $\mathscr{Q}$ be the vector bundle on $\widetilde{\mathbb{P}^{3}}$ introduced in Proposition 3.10. Let $\widetilde{\Phi}_{V_{b}}: \widetilde{\mathbb{P}^{3}} \longrightarrow$ $\mathbb{G}(b, d)$ be the morphism induced by $\mathscr{Q}$ and let $Z_{V_{a}}$ be the Schubert variety in $\mathbb{G}(b, d)$ as in Definition 3.8. Then the class of the pullback $\widetilde{\Phi}_{V_{b}}^{*}\left(\left[Z_{V_{a}}\right]\right)$ in the Chow group of $\widetilde{\mathbb{P}^{3}}$ equals

$$
\begin{array}{ll}
\mathrm{c}_{3}(\mathscr{Q}) & \text { if } a+b+1-c=3 \text { and } d-c=1, \\
\mathrm{c}_{3}(\mathscr{Q})-2 \mathrm{c}_{1}(\mathscr{Q}) \mathrm{c}_{2}(\mathscr{Q})+\mathrm{c}_{1}(\mathscr{Q})^{3} & \text { if } a+b+1-c=1 \text { and } d-c=3 .
\end{array}
$$

Proof. As we mentioned before Proposition 3.10, the statement follows from the Porteous-Giambelli formula, which states that the pullback we are interested in is given by the determinant:

$$
\left|\begin{array}{cccc}
\mathrm{c}_{(b+1)-(c-a)}(\mathscr{Q}) & \mathrm{c}_{(b+2)-(c-a)}(\mathscr{Q}) & \cdots & \mathrm{c}_{(b+d-a)-2(c-a)}(\mathscr{Q}) \\
\mathrm{c}_{b-(c-a)}(\mathscr{Q}) & \mathrm{c}_{(b+1)-(c-a)}(\mathscr{Q}) & \cdots & \mathrm{c}_{(b+d-a)-2(c-a)+1}(\mathscr{Q}) \\
\vdots & & & \\
\mathrm{c}_{b-(d-a)+2}(\mathscr{Q}) & \mathrm{c}_{b-(d-a)+3}(\mathscr{Q}) & \cdots & \mathrm{c}_{(b+1)-(c-a)}(\mathscr{Q})
\end{array}\right|
$$

In the first case we obtain $c_{3}(\mathscr{Q})$, while in the second case we get

$$
\left|\begin{array}{ccc}
\mathrm{c}_{1}(\mathscr{Q}) & \mathrm{c}_{2}(\mathscr{Q}) & \mathrm{c}_{3}(\mathscr{Q}) \\
1 & \mathrm{c}_{1}(\mathscr{Q}) & \mathrm{c}_{2}(\mathscr{Q}) \\
0 & 1 & \mathrm{c}_{1}(\mathscr{Q})
\end{array}\right|=\mathrm{c}_{1}(\mathscr{Q})^{3}+\mathrm{c}_{3}(\mathscr{Q})-2 \mathrm{c}_{1}(\mathscr{Q}) \mathrm{c}_{2}(\mathscr{Q})
$$

Proposition 3.12 computes the degrees of the cycles obtained in Proposition 3.11 in terms of the parameters $a, b, c$ and $d$ of our initial problem. The result is an application of the so-called Bott residue formula, which states the following. Suppose that $X$ is a smooth variety on which a torus $\mathbb{T}$ acts, and let $\mathscr{E}$ be a $\mathbb{T}$ equivariant vector bundle on $X$ of rank $r$. Let $p \in \mathbb{C}\left[Z_{0}, \ldots, Z_{r}\right]$ be a polynomial, 
and denote by $p(\mathscr{E})$ the expression $p\left(\mathrm{c}_{0}(\mathscr{E}), \ldots, \mathrm{c}_{r}(\mathscr{E})\right)$. Then, the degree of $p(\mathscr{E})$ can be computed by considering the fixed locus $X^{\mathbb{T}}$ of the action of $\mathbb{T}$ on $X$, namely

$$
\operatorname{deg}(p(\mathscr{E}))=\operatorname{deg} \sum_{L \subseteq X^{\mathbb{T}}} i_{*}^{L}\left(\frac{p^{\mathbb{T}}\left(\mathscr{E}_{L}\right)}{c_{d_{L}}^{\mathbb{T}}\left(\mathscr{N}_{L / X}\right)}\right),
$$

where the sum varies over the components $L$ of the fixed locus $X^{\mathbb{T}}$, the number $d_{L}$ is the codimension of $L$ in $X$, the sheaf $\mathscr{N}_{L / X}$ is the normal bundle of $L$ in $X$, each map $i^{L}$ is the canonical inclusion $L \hookrightarrow X$ and the quantity $p^{\mathbb{T}}\left(\mathscr{E}_{\mid}\right)$ is $p\left(\mathrm{c}_{0}^{\mathbb{T}}\left(\mathscr{E}_{\left.\right|_{L}}\right), \ldots, \mathrm{c}_{r}^{\mathbb{T}}\left(\mathscr{E}_{\left.\right|_{L}}\right)\right)$. Here $\mathrm{c}_{i}^{\mathbb{T}}\left(\mathscr{E}_{\left.\right|_{L}}\right)$ is the so-called $i$-th $\mathbb{T}$-equivariant Chern class of $\mathscr{E}_{L}$, and Equation (12) should be read as an equality in the $\mathbb{T}$-equivariant Chow ring of $X$. We refer to the lecture notes [MAV01], and to the references therein, for the definitions and the properties of these object.

Proposition 3.12. With the notation as in Proposition 3.11, we have

$$
\begin{aligned}
\operatorname{deg}\left(c_{1}(\mathscr{Q})^{3}+c_{3}(\mathscr{Q})-2 c_{1}(\mathscr{Q}) c_{2}(\mathscr{Q})\right) & =6\left(\begin{array}{c}
a+3 \\
3
\end{array}\right)\left(\begin{array}{c}
b+3 \\
3
\end{array}\right), \\
\operatorname{deg}\left(c_{3}(\mathscr{Q})\right) & =\frac{1}{6} a b\left(a^{2}-1\right)\left(b^{2}-1\right) .
\end{aligned}
$$

Proof. As we described before Proposition 3.10, the natural right action of $\mathbb{P G L}(2, \mathbb{C})$ on $\mathbb{P}^{3}$ determines an action of $\mathbb{P G L}(2, \mathbb{C})$ on the blowup $\widetilde{\mathbb{P}^{3}}$ and on the sheaf $\mathscr{Q}$ such that $\mathscr{Q}$ is $\mathbb{P G L}(2, \mathbb{C})$-equivariant. In particular, we have an action on $\widetilde{\mathbb{P}^{3}}$ by the torus $\mathbb{T} \cong\left(\mathbb{C}^{*}\right)^{2}$ of $2 \times 2$ invertible diagonal matrices. This action determines an action on the vector bundle $\mathscr{Q}$, making it into a $\mathbb{T}$-equivariant vector bundle. Hence we are in the situation of Bott residue formula. First of all, we compute the fixed locus of the action of $\mathbb{T}$ on $\widetilde{\mathbb{P}^{3}}$.

Lemma 3.13. The fixed locus of the action of $\mathbb{T}$ on $\widetilde{\mathbb{P}^{3}}$ is constituted of two lines and $2(b+1)(d-b)$ points.

Proof. We start with the computation of the fixed locus of the action of $\mathbb{T}$ on $\mathbb{P}^{3}$. Here, a direct computation shows that this locus is constituted of the union of the two lines $L_{1}=\{\alpha=\gamma=0\}$ and $L_{2}=\{\beta=\delta=0\}$. Then by construction the fixed locus of the action of $\mathbb{T}$ on $\widetilde{\mathbb{P}^{3}}$ is contained in the preimage $\tau^{-1}\left(L_{1} \cup L_{2}\right)$, where $\tau: \widetilde{\mathbb{P}^{3}} \longrightarrow \mathbb{P}^{3}$ is the canonical map. By continuity, the strict transforms of $L_{1}$ and $L_{2}$, which we denote by $\widetilde{L}_{1}$ and $\widetilde{L}_{2}$, are fixed by $\mathbb{T}$. In order to determine whether some other points in $\tau^{-1}\left(L_{i}\right)$ for $i \in\{1,2\}$ are fixed by $\mathbb{T}$, we can argue as in Proposition 3.10 and do the computations assuming that $\widetilde{\mathbb{P}^{3}}$ is the blowup of $\mathbb{P}^{3}$ along the line $\{\gamma=\delta=0\}$. With the same choice of coordinates as in Proposition 3.10 , the action of an element $\left(\begin{array}{cc}u & 0 \\ 0 & v\end{array}\right)$ on a point $(\alpha: \beta: \bar{\gamma}: \bar{\delta}: w)$ is given by:

$$
\left(\begin{array}{c}
\alpha \\
\beta \\
\bar{\gamma} \\
\bar{\delta} \\
w
\end{array}\right) \mapsto\left(\begin{array}{c}
\alpha u \\
\beta v \\
\bar{\gamma} u \\
\bar{\delta} v \\
w
\end{array}\right)
$$


Hence a direct computation shows that the fixed components are:

$$
\begin{array}{rlr}
\alpha=\bar{\gamma}=0, & \beta=\bar{\delta}=0, \\
w=\alpha=\bar{\delta}=0, & w=\beta=\bar{\gamma}=0 .
\end{array}
$$

Notice that the first two are the strict transforms of $L_{1}$ and $L_{2}$, while the second two are two isolated points on the exceptional divisor.

Once the fixed locus of the torus action is computed, we know from the general theory (see [LM98, Section 3.5, Theorem 32]) that the restriction of a $\mathbb{T}$-equivariant vector bundle to the fixed locus of the $\mathbb{T}$-action on the base splits as a direct sum of eigenbundles. It is crucial to compute this decomposition in order to determine the $\mathbb{T}$-equivariant Chern classes of the vector bundle. In fact, suppose that $\mathscr{E}$ is a $\mathbb{T}$-equivariant vector bundle on a smooth $\mathbb{T}$-variety $X$, and the action of $\mathbb{T}$ on $X$ is trivial, then we have a decomposition $\mathscr{E}=\bigoplus \mathscr{E}_{\chi}$ into eigenbundles, where $\chi$ varies over the characters of $\mathbb{T}$. In this situation, we can express the $\mathbb{T}$-equivariant Chern classes of each eigenbundle $\mathscr{E}_{\chi}$ as (see [EG98, Lemma 3])

$$
\mathrm{c}_{i}^{\mathbb{T}}\left(\mathscr{E}_{\chi}\right)=\sum_{j \leq i}\left(\begin{array}{c}
r-j \\
i-j
\end{array}\right) \mathrm{c}_{j}\left(\mathscr{E}_{\chi}\right) \chi^{i-j}
$$

where $r$ is the rank of $\mathscr{E}_{\chi}$. At this point Whitney sum formula, which holds also in the equivariant setting, provides the equivariant Chern classes of $\mathscr{E}$.

We therefore proceed by computing the decomposition into eigensubbundles of the restriction of the vector bundle $\mathscr{Q}$ to the various components of the fixed locus of the action of $\mathbb{T}$ on $\widetilde{\mathbb{P}^{3}}$.

Lemma 3.14. The restriction of $\mathscr{Q}$ to $\widetilde{L}_{i}$ for $i \in\{1,2\}$ splits into $b+1$ eigenbundles of rank 1 , each isomorphic to $\mathscr{O}_{\mathbb{P}^{1}}$. The corresponding characters are $u^{d}, \ldots, u^{d-b} v^{b}$ in the case of $\widetilde{L}_{2}$ and $u^{b} v^{d-b}, \ldots, v^{d}$ in the case of $\widetilde{L}_{1}$.

Proof. If $m_{0}, \ldots, m_{d}$ are the generators of the module $M$, whose sheafification is the coherent sheaf $\mathscr{M}$ on $\mathbb{P}^{3}$, then an element $\left(\begin{array}{cc}u & 0 \\ 0 & v\end{array}\right)$ sends each $m_{j}$ to $\left(u^{d-j} v^{j}\right) m_{i}$. This follows from the proof of Lemma 3.1. Let $\widetilde{m}_{0}, \ldots, \widetilde{m}_{d}$ be the generators of $Q$, the module whose sheafification is $\mathscr{Q}$, corresponding to $m_{0}, \ldots, m_{d}$. Then $\left(\begin{array}{ll}u & 0 \\ 0 & v\end{array}\right)$ sends $\widetilde{m}_{j}$ to $\left(u^{d-j} v^{j}\right) \widetilde{m}_{j}$. The restriction $\mathscr{Q}_{\tilde{L}_{1}}$ of $\mathscr{Q}$ to $\widetilde{L}_{i}$ is generated by the restrictions of the elements $\widetilde{m}_{0}, \ldots, \widetilde{m}_{d}$, and by what we have just proved these restrictions generate eigensubbundles of mutually different characters; each of the eigensubbundles is isomorphic to $\mathscr{O}_{\mathbb{P}^{1}}$ because it is generated by a single section of degree 0 . To conclude the proof, we only have to compute which of the restrictions of the elements $\widetilde{m}_{0}, \ldots, \widetilde{m}_{d}$ generate $\mathscr{Q}_{\widetilde{L}_{i}}$, and this can be checked by looking at the stalk of the vector bundle at an arbitrary point of each line.

If we pick a point $P \in \widetilde{L}_{2}$ that does not lie on the exceptional divisor, then we can do our computations in $\mathbb{P}^{3}$. So, using the left action of $\operatorname{PGL}(2, \mathbb{C})$ on $\mathbb{P}^{3}$, we can suppose that $P=\left(\begin{array}{ll}1 & 0 \\ 0 & 0\end{array}\right)$, namely $\beta=\gamma=\delta=0$. Hence we are in the situation of the proof of Proposition 3.7, and here we see that the restriction of the first $b+1$ elements $\widetilde{m}_{0}, \ldots, \widetilde{m}_{b}$ generate $\mathscr{Q}_{\widetilde{L}_{2}}$. Similarly, when $P \in \widetilde{L}_{1}$ does not lie on the exceptional divisor, an analogous version of the local analysis performed 
in Proposition 3.7 shows that $\mathscr{Q}_{\widetilde{L}_{1}}$ is generated by $\widetilde{m}_{d-b}, \ldots, \widetilde{m}_{d}$. In this case one can perform Gaussian elimination from the right to the left because the situation is "mirrored" with respect to the previous one.

Lemma 3.15. The restriction of $\mathscr{Q}$ to each of the $2(b+1)(d-b)$ fixed points splits into $b+1$ eigenbundles of rank 1 , each hence isomorphic to $\mathbb{C}$. The corresponding characters are $u^{d}, \ldots, u^{d-(b-1)} v^{b-1}, u^{d-(b+1)} v^{b+1}$ in the case of the points above $L_{2}$ and $u^{b+1} v^{d-(b+1)}, u^{b-1} v^{d-(b-1)}, \ldots, v^{d}$ in the case of the points above $L_{1}$.

Proof. As in the proof of Lemma 3.14, we know that each section $\widetilde{m}_{j}$ of $\widetilde{\mathscr{Q}}$ is sent to $\left(u^{d-j} v^{j}\right) \widetilde{m}_{j}$ by the action of the torus $\mathbb{T}$. Hence, as in Lemma 3.14 , the restriction $\widetilde{\mathscr{Q}}_{P}$, where $P$ is any of the $2(b+1)(d-b)$ fixed points, splits into the direct sum of trivial eigensubbundles; to determine the relevant characters it is enough to understand which of the restrictions to $P$ of the sections $\left\{\widetilde{m}_{j}\right\}$ do not vanish.

Suppose that $P$ is a point over $L_{2}$. Since what we need to perform is a local computation, we can put ourselves in the situation of Proposition 3.13. Then, we can take $w, \beta$ and $\bar{\gamma}$ as local coordinates for $P$, setting $\alpha=\bar{\delta}=1$. In this situation the matrix $\mathcal{Q}$ whose columns generate $\mathscr{Q}$ at $P$ has entries

$$
\frac{\partial^{d} G_{i}(s+\beta t, \bar{\gamma} w s+w t)}{\partial s^{d-j} t^{j}} .
$$

By employing the substitution $s \mapsto s-\beta t$ and setting $\widetilde{D}=\bar{\gamma} \beta-1$, the entries of the matrix become

$$
\frac{\partial^{d} G_{i}(s, \bar{\gamma} w s+w \widetilde{D} t)}{\partial s^{d-j} t^{j}} .
$$

Using the Taylor expansion already employed in Proposition 3.10, we obtain

$$
\mathcal{Q}_{i j}=\frac{(w \widetilde{D})^{j}}{j !} \cdot \frac{\partial^{j} G_{i}}{\partial t^{j}}(1, \bar{\gamma} w) .
$$

Now we can proceed with the Gaussian elimination as described in Proposition 3.10 until we reach the situation of Equation (11). From the shape of the matrix we infer that the first $b$ columns are linearly independent, and in order to prove our claim we just have to show that the $b+2$-th column gives a system of free generators for $\mathscr{Q}$ at $P$. The last row of the matrix $\mathcal{Q}$ has the following shape:

$$
\left(\begin{array}{lllll}
\underbrace{0 \ldots 0}_{b \text { zeros }} & \overline{\mathcal{Q}}_{b b} & w \widetilde{D} \cdot \overline{\mathcal{Q}}_{b, b+1} & w^{2} \widetilde{D}^{2} \cdot * & \cdots
\end{array}\right) .
$$

Here, as in Proposition 3.10, the polynomial $\overline{\mathcal{Q}}_{b b}$ is of the form $H_{b}(1, \bar{\gamma} w)$ for some polynomial $H_{b}(x, y)$ such that $\operatorname{ord}_{(1: 0)} H_{b}=1$, while $\overline{\mathcal{Q}}_{b, b+1}$ is of the form $H_{b+1}(1, \bar{\gamma} w)$, where $\operatorname{ord}_{(1: 0)} H_{b+1}=0$. This implies that $\overline{\mathcal{Q}}_{b b}=\bar{\gamma} w+\ldots$, while $\overline{\mathcal{Q}}_{b, b+1}$ is invertible in the local ring at $P$. Since also $\widetilde{D}$ is invertible in that ring, it follows that the ideal generated by the entries of the last row of $\mathcal{Q}$ is $(w)$, and it is generated by $w \widetilde{D} \cdot \overline{\mathcal{Q}}_{b, b+1}$. This implies that the sections that do not vanish at $P$ are $\widetilde{m}_{0}, \ldots, \widetilde{m}_{b-1}$ and $\tilde{m}_{b+1}$, showing our claim.

The case of points over $L_{1}$ is similar, as discussed in Lemma 3.14. 
Lemma 3.16. For $k \in\{1,2,3\}$ let $\varsigma_{k}\left(y_{0}, \ldots, y_{t}\right)$ be the $k$-th elementary symmetric polynomial in the variables $y_{0}, \ldots, y_{t}$. The $k$-th equivariant Chern classes of the restriction of $\mathscr{Q}$ to $\widetilde{L}_{i}$ for $i \in\{1,2\}$ are

$$
\begin{aligned}
& \mathrm{c}_{k}^{\mathbb{T}}\left(\mathscr{Q}_{\tilde{L}_{1}}\right)=\varsigma_{k}((d-i) v+i u, \text { for } i \in\{0, \ldots, b\}), \\
& \mathrm{c}_{k}^{\mathbb{T}}\left(\mathscr{Q}_{\tilde{L}_{2}}\right)=\varsigma_{k}((d-i) u+i v, \text { for } i \in\{0, \ldots, b\}) .
\end{aligned}
$$

Proof. For each eigensubbundle $\mathscr{E}_{\chi}$ of $\mathscr{Q}_{\tilde{L}_{i}}$ of character $\chi$ we have $c_{0}\left(\mathscr{E}_{\chi}\right)=1$ and $\mathrm{c}_{i}\left(\mathscr{E}_{\chi}\right)=0$ for all $i \geq 1$. In fact, by Lemma 3.14 every subbundle $\mathscr{E}_{\chi}$ is isomorphic to $\mathscr{O}_{\mathbb{P}^{1}}$. Hence, by Equation $(13)$ we have $c_{1}^{\mathbb{T}}\left(\mathscr{E}_{\chi}\right)=\chi$ and $\mathrm{c}_{i}^{\mathbb{T}}(\mathscr{E})=0$ for $i \geq 2$. For example, if we consider $\mathscr{Q}_{\tilde{L}_{2}}$ and we take $\chi=u^{d-i} v^{i}$, namely $\mathscr{E}_{\chi}$ is the eigensubbundle generated by the restriction to $\widetilde{L}_{2}$ of the global section $\widetilde{m}_{i}$, then we have $\mathrm{c}_{1}^{\mathbb{T}}\left(\mathscr{E}_{\chi}\right)=(d-i) u+i v$ (here the character is reported in logarithmic notation). The statement then follows from the Whitney sum formula for the Chern class of a direct sum and the description of the characters of the eigensubbundles provided by Lemma 3.14 .

Lemma 3.17. For $k \in\{1,2,3\}$ let $\varsigma_{k}\left(y_{0}, \ldots, y_{t}\right)$ be the $k$-th elementary symmetric polynomial in the variables $y_{0}, \ldots, y_{t}$. The $k$-th equivariant Chern classes of the restriction of $\mathscr{Q}$ to each of the $2(b+1)(d-b)$ fixed points are

$$
\mathrm{c}_{k}^{\mathbb{T}}\left(\mathscr{Q}_{P}\right)=\varsigma_{k}\left(\begin{array}{c}
(d-i) v+i u, \\
i \in\{0, \ldots, b-1\}
\end{array},(d-(b+1)) v+(b+1) u\right)
$$

in the case of the points $P$ over $L_{1}$, and

$$
c_{k}^{\mathbb{T}}\left(\mathscr{Q}_{P}\right)=\varsigma_{k}\left(\begin{array}{c}
(d-i) u+i v, \\
i \in\{0, \ldots, b-1\}
\end{array},(d-(b+1)) u+(b+1) v\right)
$$

in the case of the points $P$ over $L_{2}$.

Proof. The proof is analogous to the one of Lemma 3.16.

Lemma 3.18. The normal bundle of $\widetilde{L}_{1}$ (respectively, $\widetilde{L}_{2}$ ) in $\widetilde{\mathbb{P}^{3}}$ is an eigenbundle of character $u / v$ (respectively, $v / u$ ). The Chern polynomial of $\mathscr{N}_{\widetilde{L}_{i} / \widetilde{\mathbb{P}^{3}}}$ is $1+(2-(b+1)(d-b)) h$, where $h$ is the class of a point, for both $i=1$ and $i=2$.

Proof. We prove the statement for $\widetilde{L}_{1}$, the argument for $\widetilde{L}_{2}$ is analogous. We start by showing that the whole bundle $\mathscr{N}_{\widetilde{L}_{1} / \widetilde{\mathbb{P}^{3}}}$ is an eigenbundle of character $u / v$. It suffices to show this locally at a point, since the decomposition into eigensubbundles is canonical. Consider hence a point $P=(0: \beta: 0: \bar{\delta}: w)$ on $\widetilde{L}_{1}$ (here we use the notation as in Lemma 3.13). We can pick affine coordinates $\alpha, \bar{\gamma}$, and $w$ for $P$, setting $\beta=\bar{\delta}=1$. In these local coordinates, the action of $\mathbb{T}$ is given by

$$
\left(\begin{array}{c}
\alpha \\
\bar{\gamma} \\
w
\end{array}\right) \mapsto\left(\begin{array}{c}
u / v \alpha \\
u / v \bar{\delta} \\
w
\end{array}\right)
$$


In fact, recall that the action of $\mathbb{T}$ on the coordinates $\alpha, \beta, \bar{\gamma}, \bar{\delta}, w$ is

$$
\left(\begin{array}{c}
\alpha \\
1 \\
\bar{\gamma} \\
1 \\
w
\end{array}\right) \quad \mapsto\left(\begin{array}{c}
u \alpha \\
v \\
u \bar{\gamma} \\
v \\
w
\end{array}\right)=\left(\begin{array}{c}
u / v \alpha \\
1 \\
u / v \bar{\gamma} \\
1 \\
w
\end{array}\right)
$$

where we use the special grading of the coordinates for the equality on the right. Because of the choice of coordinates, and recalling that $\widetilde{L}_{1}$ is defined by $\alpha=\bar{\gamma}=0$, we can write

$$
\left(\mathscr{N}_{\widetilde{L}_{1} / \widetilde{\mathbb{P}^{3}}}\right)_{P} \cong \frac{\left\langle\partial_{\alpha}, \partial_{\bar{\gamma}}, \partial_{w}\right\rangle}{\left\langle\partial_{w}\right\rangle}
$$

Since the action on the tangent space at $P$ of $\widetilde{\mathbb{P}^{3}}$ is given by the Jacobian of Equation (14), and because of the description of the normal bundle in Equation (15) we see that the action on the normal bundle is given by the first principal $2 \times 2$ minor of that Jacobian, which is a diagonal matrix with $u / v$ as diagonal entries. Hence the whole $\mathscr{N}_{\widetilde{L}_{1} / \widetilde{\mathbb{P}^{3}}}$ is an eigenbundle of character $u / v$.

Let us now compute the Chern polynomial of the normal bundle of $\widetilde{L}_{1}$; the same proof works for $\widetilde{L}_{2}$. We write $L$ for $L_{1}$. Since both $L$ and $B$ (the blowup center) are regularly embedded in $\mathbb{P}^{3}$, and the intersection $L \cap B$ is regularly embedded in both $L$ and $B$, we can use the result of Aluffi [Alu10, Section 4.3] to compute the Chern polynomial $c\left(\mathscr{N}_{\widetilde{L} / \widetilde{\mathbb{P}^{3}}}\right)$. In fact, both $L$ and $B$ are contained in the smooth quadric $Q=\{\alpha \delta-\beta \gamma=0\}$, and inside $Q$ they intersect properly, since their intersection is equidimensional of codimension 2 in $Q$. If $\widetilde{Q}$ is the strict transform of $Q$ in $\widetilde{\mathbb{P}^{3}}$, and $\tau: \widetilde{\mathbb{P}^{3}} \longrightarrow \mathbb{P}^{3}$ denotes the blow down map, and $i_{\widetilde{L}}: \widetilde{L} \hookrightarrow \widetilde{Q}$ and $i_{\widetilde{Q}}: \widetilde{Q} \hookrightarrow \widetilde{\mathbb{P}^{3}}$ denote the closed immersions, then

$$
c\left(\mathscr{N}_{\widetilde{L} / \widetilde{\mathbb{P}^{3}}}\right)=\tau_{\left.\right|_{\widetilde{L}} ^{*}}^{*} c\left(\mathscr{N}_{L / Q}\right) \cdot i_{\widetilde{L}}^{*} c\left(\tau_{\left.\right|_{\widetilde{Q}}}^{*} \mathscr{N}_{Q / \mathbb{P}^{3}} \otimes i_{\widetilde{Q}}^{*} \mathscr{O}_{\widetilde{\mathbb{P}^{3}}}(-E)\right) .
$$

The formula before can be written as

$$
c\left(\mathscr{N}_{\widetilde{L} / \widetilde{\mathbb{P}^{3}}}\right)=c\left(\mathscr{N}_{L / Q}\right) \cdot c\left(\mathscr{N}_{Q / \mathbb{P}^{3}} \otimes \mathscr{O}_{\widetilde{\mathbb{P}^{3}}}(-E)\right)
$$

if we omit the pullbacks. Since by [Har77, Example V.1.4.1] the degree of $\mathscr{N}_{L / Q}$ equals the self-intersection of $L$ inside $Q$, we have $\operatorname{deg}\left(\mathscr{N}_{L / Q}\right)=0$ and so $c\left(\mathscr{N}_{L / Q}\right)$ equals 1. By $\left[\right.$ GH78, Adjunction Formula I] we have $\mathscr{N}_{Q / \mathbb{P} 3} \cong \mathscr{O}_{\mathbb{P}^{3}}(Q)_{\mid Q}$ and so, as a sheaf on $\mathbb{P}^{1} \times \mathbb{P}^{1}$, the latter is $\mathscr{O}_{\mathbb{P}^{1} \times \mathbb{P}^{1}}(2,2)$. Since $\tau_{\left.\right|_{\tilde{Q}}}$ is an isomorphism (in fact the blowup center $B$ is a divisor in $Q)$, we can compute $\tau_{\left.\right|_{\tilde{Q}}}^{*} \mathscr{N}_{Q / \mathbb{P}^{3}} \otimes i_{\widetilde{Q}}^{*} \mathscr{O}_{\widetilde{\mathbb{P}^{3}}}(-E)$ as a sheaf on $\mathbb{P}^{1} \times \mathbb{P}^{1}$; this amounts to shift $\mathscr{N}_{Q / \mathbb{P}^{3}}$ by $\mathscr{O}_{Q}(-B)$, obtaining $\mathscr{O}_{\mathbb{P}^{1} \times \mathbb{P}^{1}}(2,2-K)$, where $K=(b+1)(d-b)$. Notice that here we used that the class in $\mathbb{P}^{1} \times \mathbb{P}^{1}$ of the lines in $B$ is $(0,1)$, thus the class of $L$ is $(1,0)$. Eventually, restricting this bundle to $\widetilde{L}$ gives $\mathscr{O}_{\widetilde{L}}(2-K)$, because the intersection product in $\mathbb{P}^{1} \times \mathbb{P}^{1}$ of the classes $(1,0)$ and $(2,2-K)$ equals $2-K$. Hence we obtain

$$
c\left(\mathscr{N}_{\widetilde{L} / \widetilde{\mathbb{P}^{3}}}\right)=1+(2-(b+1)(d-b)) h,
$$

where $h$ is the class of a point. 
Lemma 3.19. The normal bundle - namely, the tangent space - of each of the $2(b+1)(d-b)$ fixed points splits as the sum of two eigenbundles as follows:

- one of rank 1 of character $v / u$ and another of rank 2 of character $u / v$ for the points over $L_{1}$;

- one of rank 1 of character $u / v$ and another of rank 2 of character $v / u$ for the points over $L_{2}$.

Proof. Let $P$ be a point over $L_{1}$. We compute the action of the torus locally around $P$. Thus we can suppose that we are in the setting of Lemma 3.13, namely $P$ has equations $w=\alpha=\bar{\delta}=0$. Similarly as what we did in Lemma 3.18, we can pick affine coordinates $w, \alpha, \bar{\delta}$ for $P$, setting $\beta=\bar{\gamma}=1$. In these local coordinates, the action of $\mathbb{T}$ is given by

$$
\left(\begin{array}{c}
\alpha \\
\bar{\delta} \\
w
\end{array}\right) \mapsto\left(\begin{array}{c}
u / v \alpha \\
v / u \bar{\delta} \\
u / v w
\end{array}\right)
$$

because the action of $\mathbb{T}$ on the coordinates $\alpha, \beta, \bar{\gamma}, \bar{\delta}, w$ is

$$
\left(\begin{array}{c}
\alpha \\
1 \\
1 \\
\bar{\delta} \\
w
\end{array}\right) \mapsto\left(\begin{array}{c}
u \alpha \\
v \\
u \\
v \bar{\delta} \\
w
\end{array}\right)=\left(\begin{array}{c}
\alpha \\
v / u \\
1 \\
v / u \bar{\delta} \\
w
\end{array}\right)=\left(\begin{array}{c}
u / v \alpha \\
1 \\
1 \\
v / u \bar{\delta} \\
u / v w
\end{array}\right),
$$

where we use the special grading of the coordinates for the equalities on the right. Since the action on the tangent bundle is given by the Jacobian of the action in Equation (16), we obtain two eigensubbundles of the desired rank and character.

The argument when $P$ is a point over $L_{2}$ is identical.

Lemma 3.20. The second equivariant Chern class of the normal bundle of $\widetilde{L}_{i}$ is

$$
\begin{aligned}
& \mathrm{c}_{2}^{\mathbb{T}}\left(\mathscr{N}_{\widetilde{L}_{1} / \widetilde{\mathbb{P}^{3}}}\right)=(u-v)^{2}+(u-v)(2-(b+1)(d-b)) h, \\
& \mathrm{c}_{2}^{\mathbb{T}}\left(\mathscr{N}_{\widetilde{L}_{2} / \widetilde{\mathbb{P}^{3}}}\right)=(v-u)^{2}+(v-u)(2-(b+1)(d-b)) h .
\end{aligned}
$$

Proof. We argue as in Lemma 3.16 using the results of Lemma 3.18.

Lemma 3.21. The third equivariant Chern class of the normal bundle of each of the $2(b+1)(d-b)$ fixed points is

$$
\begin{aligned}
\mathrm{c}_{3}^{\mathbb{T}}\left(\mathscr{N}_{P / \widetilde{\mathbb{P}^{3}}}\right) & =(v-u)^{3} \quad \text { for points } P \text { above } L_{1} \text { and } \\
\mathrm{c}_{3}^{\mathbb{T}}\left(\mathscr{N}_{P / \widetilde{\mathbb{P}^{3}}}\right) & =(u-v)^{3} \quad \text { for points } P \text { above } L_{2} .
\end{aligned}
$$

Proof. Suppose that $P$ is a point over $L_{2}$, then by Lemma 3.19 we have that $\mathscr{N}_{P / \widetilde{\mathbb{P}^{3}}} \cong E_{1} \oplus E_{2}$ where $E_{i}$ are vector spaces of dimension $i$ and of characters $u / v$ and $v / u$, respectively. Using [EG98, Lemma 3] we find that $\mathrm{c}_{1}^{\mathbb{T}}\left(E_{1}\right)=u-v$, while $\mathrm{c}_{i}^{\mathbb{T}}\left(E_{1}\right)=0$ for all $i \geq 2$, and $\mathrm{c}_{2}^{\mathbb{T}}\left(E_{2}\right)=(v-u)^{2}$, while $\mathrm{c}_{i}^{\mathbb{T}}\left(E_{1}\right)=0$ for all $i \geq 3$. Hence $\mathrm{c}_{3}^{\mathbb{T}}\left(\mathscr{N}_{P / \widetilde{\mathbb{P}^{3}}}\right)=\mathrm{c}_{1}^{\mathbb{T}}\left(E_{1}\right) \mathrm{c}_{2}^{\mathbb{T}}\left(E_{2}\right)=(u-v)^{3}$ by the Whitney formula.

The proof for points over $L_{1}$ is analogous. 
We can now finally use Bott residue formula to prove Proposition 3.12. We only present the computation of $\operatorname{deg}\left(c_{3}(\mathscr{Q})\right)$ : the other case is analogous, but the computations are more tedious. Equation (12) gives

$$
\begin{aligned}
& \operatorname{deg}\left(\mathrm{c}_{3}(\mathscr{Q})\right)=\operatorname{deg} i_{*}^{\widetilde{L}_{1}}\left(\frac{\mathrm{c}_{3}^{\mathbb{T}}\left(\mathscr{Q}_{\left.\right|_{\tilde{L}_{1}}}\right)}{\mathrm{c}_{2}^{\mathbb{T}}\left(\mathscr{N}_{\widetilde{L}_{1} / \widetilde{\mathbb{P}^{3}}}\right)}\right)+\operatorname{deg} i_{*}^{\widetilde{L}_{2}}\left(\frac{\mathrm{c}_{3}^{\mathbb{T}}\left(\mathscr{Q}_{\left.\right|_{\tilde{L}_{2}}}\right)}{\mathrm{c}_{2}^{\mathbb{T}}\left(\mathscr{N}_{\widetilde{L}_{2} / \mathbb{P}^{3}}\right)}\right) \\
& +(b+1)(d-b) \underbrace{\operatorname{deg} i_{*}^{P_{1}}\left(\frac{\mathrm{c}_{3}^{\mathbb{T}}\left(\mathscr{Q}_{\left.\right|_{1}}\right)}{\mathrm{c}_{3}^{\mathbb{T}}\left(\mathscr{N}_{P_{1} / \widetilde{\mathbb{P}^{3}}}\right)}\right)}_{\text {a point over } L_{1}}+(b+1)(d-b) \underbrace{\operatorname{deg} i_{*}^{P_{2}}\left(\frac{\mathrm{c}_{3}^{\mathbb{T}}\left(\mathscr{Q}_{\left.\right|_{P_{2}}}\right)}{\mathrm{c}_{3}^{\mathbb{T}}\left(\mathscr{N}_{P_{2} / \widetilde{\mathbb{P}^{3}}}\right)}\right)}_{\text {a point over } L_{2}} .
\end{aligned}
$$

The previous results allow us to compute each of the four summands:

$$
\begin{aligned}
\operatorname{deg} i_{*}^{\widetilde{L}_{1}}\left(\frac{c_{3}^{\mathbb{T}}\left(\mathscr{Q}_{\widetilde{L}_{1}}\right)}{c_{2}^{\mathbb{T}}\left(\mathscr{N}_{\widetilde{L}_{1} / \widetilde{P}^{3}}\right)}\right)=\operatorname{deg} \frac{\varsigma_{3}((d-i) v+i u, i \in\{0, \ldots, b\})}{(u-v)^{2}+(u-v)(2-(b+1)(d-b)) h} \\
=\frac{\varsigma_{3}((d-i) v+i u, i \in\{0, \ldots, b\})}{u-v} \operatorname{deg} \frac{(u-v)-(2-(b+1)(d-b)) h}{(u-v)^{2}} \\
=\frac{\varsigma_{3}((d-i) v+i u, i \in\{0, \ldots, b\})(-2+(b+1)(d-b))}{(u-v)^{3}},
\end{aligned}
$$

(where in the second equality we multiplied both numerator and denominator by $(u-v)-(2-(b+1)(d-b)) h$, and we used the fact that $h^{2}=0$ in the Chow ring of $\left.\widetilde{L}_{1}\right)$

$\operatorname{deg} i_{*}^{\widetilde{L}_{2}}\left(\frac{\mathrm{c}_{3}^{\mathbb{T}}\left(\mathscr{Q}_{\left.\right|_{\tilde{L}_{2}}}\right)}{\mathrm{c}_{2}^{\mathbb{T}}\left(\mathscr{N}_{\widetilde{L}_{2} / \widetilde{\mathbb{P}^{3}}}\right)}\right)=\frac{\varsigma_{3}((d-i) u+i v, i \in\{0, \ldots, b\})(-2+(b+1)(d-b))}{(v-u)^{3}}$, $\operatorname{deg} i_{*}^{P_{1}}\left(\frac{\mathrm{c}_{3}^{\mathbb{T}}\left(\mathscr{Q}_{P_{1}}\right)}{\mathrm{c}_{3}^{\mathbb{T}}\left(\mathscr{N}_{P_{1} / \widetilde{\mathbb{P}^{3}}}\right)}\right)=\frac{1}{(v-u)^{3}} \varsigma_{3}\left(\begin{array}{c}(d-i) v+i u, \\ i \in\{0, \ldots, b-1\}\end{array},(d-(b+1)) v+(b+1) u\right)$, $\operatorname{deg} i_{*}^{P_{2}}\left(\frac{\mathrm{c}_{3}^{\mathbb{T}}\left(\mathscr{Q}_{P_{2}}\right)}{\mathrm{c}_{3}^{\mathbb{T}}\left(\mathscr{N}_{P_{2} / \widetilde{\mathbb{P}^{3}}}\right)}\right)=\frac{1}{(u-v)^{3}} \varsigma_{3}\left(\begin{array}{c}(d-i) u+i v, \\ i \in\{0, \ldots, b-1\}\end{array},(d-(b+1)) u+(b+1) v\right)$.

Hence in this way we have expressed $\operatorname{deg}\left(\mathrm{c}_{3}(\mathscr{Q})\right)$ as a rational function in $u$ and $v$. Therefore this rational function is constant, and so we can assign arbitrary values to $u$ and $v$ (as long as fractions have non-zero denominator), and we will obtain the same number. We take $u=1$ and $v=0$, and we write $K$ for the quantity $(b+1)(d-b)$. In this way, we get

$$
\begin{aligned}
\operatorname{deg}\left(c_{3}(\mathscr{Q})\right)= & (K-2) \varsigma_{3}(i, \text { for } i \in\{0, \ldots, b\}) \\
- & (K-2) \varsigma_{3}(d-i, \text { for } i \in\{0, \ldots, b\}) \\
- & K \varsigma_{3}(i, \text { for } i \in\{0, \ldots, b-1\},(b+1)) \\
+ & K \varsigma_{3}(d-i, \text { for } i \in\{0, \ldots, b-1\},(d-(b+1))) .
\end{aligned}
$$


The previous formula can be simplified further, noticing that

$$
\begin{aligned}
& \varsigma_{3}(i, \text { for } i \in\{0, \ldots, b-1\},(b+1))= \\
& \varsigma_{3}(i, \text { for } i \in\{0, \ldots, b\})+\varsigma_{2}(i, \text { for } i \in\{0, \ldots, b-1\}) .
\end{aligned}
$$

We get

$$
\begin{aligned}
\operatorname{deg}\left(c_{3}(\mathscr{Q})\right)= & -2 \varsigma_{3}(i, \text { for } i \in\{0, \ldots, b\}) \\
& +2 \varsigma_{3}(d-i, \text { for } i \in\{0, \ldots, b\}) \\
& -K \varsigma_{2}(i, \text { for } i \in\{0, \ldots, b-1\}) \\
& -K \varsigma_{2}(d-i, \text { for } i \in\{0, \ldots, b-1\}) .
\end{aligned}
$$

If we write

$$
\varsigma_{3}(i, \text { for } i \in\{0, \ldots, b\})=\sum_{h=0}^{b} \sum_{l=h+1}^{b} \sum_{m=l+1}^{b} h l m,
$$

and similarly for the other summands, then we obtain the statement using standard techniques in summation or a symbolic summation software as, for example, Mathematica. We thank Christoph Koutschan for helping us with this symbolic summation problem.

Proposition 3.11 and 3.12 imply:

Theorem 3.22. Let $V_{a}$ and $V_{b}$ be general vector subspaces of $\mathbb{C}[s, t]_{d}$ of dimension $a+1$ and $b+1$, respectively. Suppose that

$$
(a+b-c+1)(d-c)=3 .
$$

Then, the cardinality of the set

$$
\left\{\sigma \in \mathbb{P G L}(2, \mathbb{C}): \operatorname{dim}\left(V_{a}+V_{b}^{\sigma}\right) \leq c+1\right\}
$$

is

$$
\begin{array}{ll}
\frac{1}{6} a b\left(a^{2}-1\right)\left(b^{2}-1\right) & \text { if } a+b+1-c=3 \text { and } d-c=1, \\
6\left(\begin{array}{c}
a+3 \\
3
\end{array}\right)\left(\begin{array}{c}
b+3 \\
3
\end{array}\right) & \text { if } a+b+1-c=1 \text { and } d-c=3 .
\end{array}
$$

Remark 3.23. Here is a funny example: let $a=1, b=0, c=1$ and $d=4$. Then we are counting the number of changes of variables such that a given general binary quartic $G$ becomes an element of a given general linear pencil $\Gamma:=\left\langle F_{0}, F_{1}\right\rangle$ of binary quartics. Our formula gives the answer 24. However, by applying a change of variables to $G$ we can only get 6 projectively different elements of $\Gamma$. In fact, binary quartics have one invariant $I$ which is of degree 6 in the coefficients (see [Dol03, Section 10.2]). Therefore the value of $I$ coincides with $I(G)$ at exactly 6 elements in the pencil, obtained by solving the equation $I\left(\lambda F_{0}+\mu F_{1}\right)=I(G)$ for $(\lambda: \mu) \in \mathbb{P}^{1}$. The discrepancy between the number of changes of variables and the number of elements in $\Gamma$ is explained by the fact that a general quartic binary form has 4 automorphisms. 
We conclude by translating the Theorem 3.22 into the answer to our initial problem. First of all, notice that if $V_{a}$ and $V_{b}$ are general and Equation $(*)$ holds, then

$$
\left\{\begin{array}{c}
\sigma \in \mathbb{P G L}(2, \mathbb{C}) \text { such that } \\
\operatorname{dim}\left(V_{a}+V_{b}^{\sigma}\right) \leq c+1
\end{array}\right\}=\left\{\begin{array}{c}
\sigma \in \mathbb{P G L}(2, \mathbb{C}) \text { such that } \\
\operatorname{dim}\left(V_{a}+V_{b}^{\sigma}\right)=c+1
\end{array}\right\} .
$$

In fact, the arguments of Section 2 show that the set of $\sigma \in \mathbb{P G L}(2, \mathbb{C})$ such that $\operatorname{dim}\left(V_{a}+V_{b}^{\sigma}\right) \leq c$ is empty. This holds because, using the notation introduced there, in this case the incidence variety $\mathscr{I}$ has codimension $(d-c)(a+b-c+2)$ in $\mathbb{G}(a, d)$, so its dimension is strictly smaller than the dimension of $\mathbb{G}(a, d) \times \mathbb{G}(b, d)$ and therefore the map $\psi$ cannot be dominant.

The two subspaces $V_{a}$ and $V_{b}$ define parametrizations $f_{a}: \mathbb{P}^{1} \longrightarrow C_{a} \subseteq \mathbb{P}^{a}$ and $f_{b}: \mathbb{P}^{1} \longrightarrow C_{b} \subseteq \mathbb{P}^{b}$. Consider the equivalence relation on triples $\left(\pi_{a}, \pi_{b}, C_{c}\right)$, where $C_{c} \subseteq \mathbb{P}^{c}$ is a rational curve of degree $d$ and $\pi_{u}: \mathbb{P}^{c} \rightarrow \mathbb{P}^{u}$ are linear projections such that $\pi_{u}\left(C_{c}\right)=C_{u}$ for $u \in\{a, b\}$, given by the action of $\mathbb{P G L}(c+1, \mathbb{C})$ :

$$
\left(\pi_{a}, \pi_{b}, C_{c}\right) \sim\left(\pi_{a} \circ \alpha, \pi_{b} \circ \alpha, \alpha^{-1}\left(C_{c}\right)\right) \quad \text { for every } \alpha \in \mathbb{P G L}(c+1, \mathbb{C}) .
$$

We now show that there is a bijection

$$
\left\{\begin{array}{c}
\sigma \in \mathbb{P G L}(2, \mathbb{C}) \text { such that } \\
\operatorname{dim}\left(V_{a}+V_{b}^{\sigma}\right)=c+1
\end{array}\right\} \longleftrightarrow\left\{\begin{array}{c}
\text { equivalence classes under } \sim \\
\text { of triples }\left(\pi_{a}, \pi_{b}, C_{c}\right)
\end{array}\right\}
$$

Starting from $\sigma \in \mathbb{P G L}(2, \mathbb{C})$, we define $f_{c}: \mathbb{P}^{1} \longrightarrow \mathbb{P}^{c}$ as the map associated to the $(c+1)$-dimensional vector space $V_{a}+V_{b}^{\sigma}$. Since $V_{a}$ and $V_{b}^{\sigma}$ are subspaces, we get projections $\pi_{a}$ and $\pi_{b}$ sending the image $C_{c}=f_{c}\left(\mathbb{P}^{1}\right)$ to $C_{a}$ and $C_{b}$, respectively. Conversely, starting from a triple $\left(\pi_{a}, \pi_{b}, C_{c}\right)$ as above, one defines $f_{c}: \mathbb{P}^{1} \longrightarrow \mathbb{P}^{c}$ as $\left(\pi_{\left.a\right|_{C_{c}}}\right)^{-1} \circ f_{a}$. Then we define $\sigma: \mathbb{P}^{1} \longrightarrow \mathbb{P}^{1}$ as $f_{b}^{-1} \circ \pi_{b} \circ f_{c}$. Here we use that $\pi_{\left.a\right|_{C_{c}}}$ and $\pi_{\left.a\right|_{C_{c}}}$ are birational because they preserve the degree of the curve. If we replace the triple $\left(\pi_{a}, \pi_{b}, C_{c}\right)$ by an equivalent one, then we get the same $\sigma$. One can also check that these two constructions are each other's inverse. Hence it follows:

Theorem 3.24. Let $C_{a} \subseteq \mathbb{P}^{a}$ and $C_{b} \subseteq \mathbb{P}^{b}$ be two general rational curves of degree $d$. Let $c$ be a natural number and suppose that the following holds:

$$
(a+b+1-c)(d-c)=3 .
$$

Then there are, up to automorphisms of $\mathbb{P}^{c}$, finitely many rational non-degenerate curves $C_{c} \subseteq \mathbb{P}^{c}$ of degree d together with linear projections $\pi_{a}: C_{c} \longrightarrow C_{a}$ and $\pi_{b}: C_{c} \longrightarrow C_{b}$.

(1) Suppose that $a+b+1-c=1$ and $d-c=3$. Then, the number of these curves and projections is

$$
\frac{1}{6}(a+3)(a+2)(a+1)(b+3)(b+2)(b+1) .
$$

(2) Suppose that $a+b+1-c=3$ and $d-c=1$. Then, the number of these curves and projections is

$$
\frac{1}{6} a b\left(a^{2}-1\right)\left(b^{2}-1\right) .
$$




\section{ACKNOWLEDGMENTS}

We thank Hoon Hong for many useful discussions that inspired this work, and Niels Lubbes for helping us with a particular case of our problem and for useful comments. We thank Christoph Koutschan for providing us the proof of a useful lemma. Matteo Gallet would like to thank Dario Portelli for teaching him, among many other things, important notions concerning intersection theory.

\section{REFERENCES}

[Alu10] P. Aluffi, Chern classes of blow-ups, Mathematical Proceedings of the Cambridge Philosophical Society 148 (2010), no. 2, 227-242.

[Arr96] E. Arrondo, Subvarieties of Grassmannians, Lecture Note Series Dipartimento di Matematica Univ. Trento 10 (1996), Available at http://www.mat.ucm.es/ arrondo/trento.pdf.

[Arr10] Wector bundles in Algebraic Geometry, Notes of the course taught at the First Summer School on Complex Geometry (Villarrica, Chile 7-9 December 2010). Available at http://www.mat.ucm.es/ arrondo/curso-chile.pdf, 2010.

[BD10] A. Bostan and P. Dumas, Wronskians and linear independence, American Mathematical Monthly 117 (2010), no. 8, 722-727.

[BKH13] J. M. Burdis, I. A. Kogan, and H. Hong, Object-image correspondence for algebraic curves under projections, SIGMA 9 (2013), 1-31.

[Cut04] S. D. Cutkosky, Resolution of singularities, Graduate Studies in Mathematics, vol. 63, American Mathematical Society, 2004.

[Dol03] I. V. Dolgachev, Lectures on invariant theory, London Mathematical Society Lecture Note Series, vol. 296, Cambridge University Press, Cambridge, 2003.

[Dol12] Classical algebraic geometry. A modern view, Cambridge University Press, Cambridge, 2012.

[EG98] D. Edidin and W. Graham, Localization in equivariant intersection theory and the Bott residue formula, American Journal of Mathematics 120 (1998), no. 3, 619-636.

[EH16] D. Eisenbud and J. Harris, 3264 and All That. A Second Course in Algebraic Geometry, Cambridge University Press, 2016.

[GH78] P. Griffiths and J. Harris, Principles of algebraic geometry, Wiley-Interscience, New York, 1978.

[Har77] R. Hartshorne, Algebraic geometry, Graduate Texts in Mathematics, vol. 52, SpringerVerlag, 1977.

[Har95] J. Harris, Algebraic geometry. A first course, Graduate Texts in Mathematics, vol. 133, Springer-Verlag, New York, 1995.

[HZ04] R. I. Hartley and A. Zisserman, Multiple View Geometry in Computer Vision, second ed., Cambridge University Press, 2004.

[KL74] G. Kempf and D. Laksov, The determinantal formula of Schubert calculus, Acta Mathematica 132 (1974), no. 1, 153-162.

[Kle74] S. L. Kleiman, The transversality of a general translate, Compositio Mathematica 28 (1974), 287-297.

[LM98] G. Luke and A.S. Mishchenko, Vector bundles and their applications, Mathematics and its Applications, vol. 447, Kluwer Academic Publishers, Dordrecht, 1998.

[MAV01] A. L. Meireles Araújo and I. Vainsencher, Equivariant intersection theory and Bott's residue formula, Matemática Contemporânea 20 (2001), 1-70.

[Sha13] I. R. Shafarevich, Basic algebraic geometry 1. Varieties in projective space, third ed., Springer, 2013. 
(Matteo Gallet) International School for Advanced Studies/Scuola Internazionale Superiore di Studi Avanzati (ISAS/SISSA), Via Bonomea 265, 34136 Trieste, Italy

Email address: mgallet@sissa.it

(Josef Schicho) Research Institute for Symbolic Computation (RISC), Johannes Kepler UNIVERSITY, LINZ

Email address: jschicho@risc.jku.at 